\title{
The iClick reaction of a BODIPY platinum(II) azido complex with electron-poor alkynes provides triazolate complexes with $\operatorname{good}^{1} \mathrm{O}_{2}$ sensitization efficiency
}

\author{
Kun Peng, ${ }^{a}$ Richard Einsele, ${ }^{a}$ Peter Irmler, ${ }^{b}$ Rainer F. Winter, ${ }^{*, b}$ and \\ Ulrich Schatzschneider ${ }^{*}$,a
}

\footnotetext{
${ }^{a}$ Institut für Anorganische Chemie, Julius-Maximilians-Universität Würzburg, Am Hubland, 97074 Würzburg, Germany

${ }^{\mathrm{b}}$ Fachbereich Chemie, Universität Konstanz, Universitätsstr. 10, 78457 Konstanz, Germany
}

*rainer.winter@uni-konstanz.de (R.W.), ulrich.schatzschneider@uni-wuerzburg.de (U.S.)

Supporting Information 
Section S1. Kinetic data

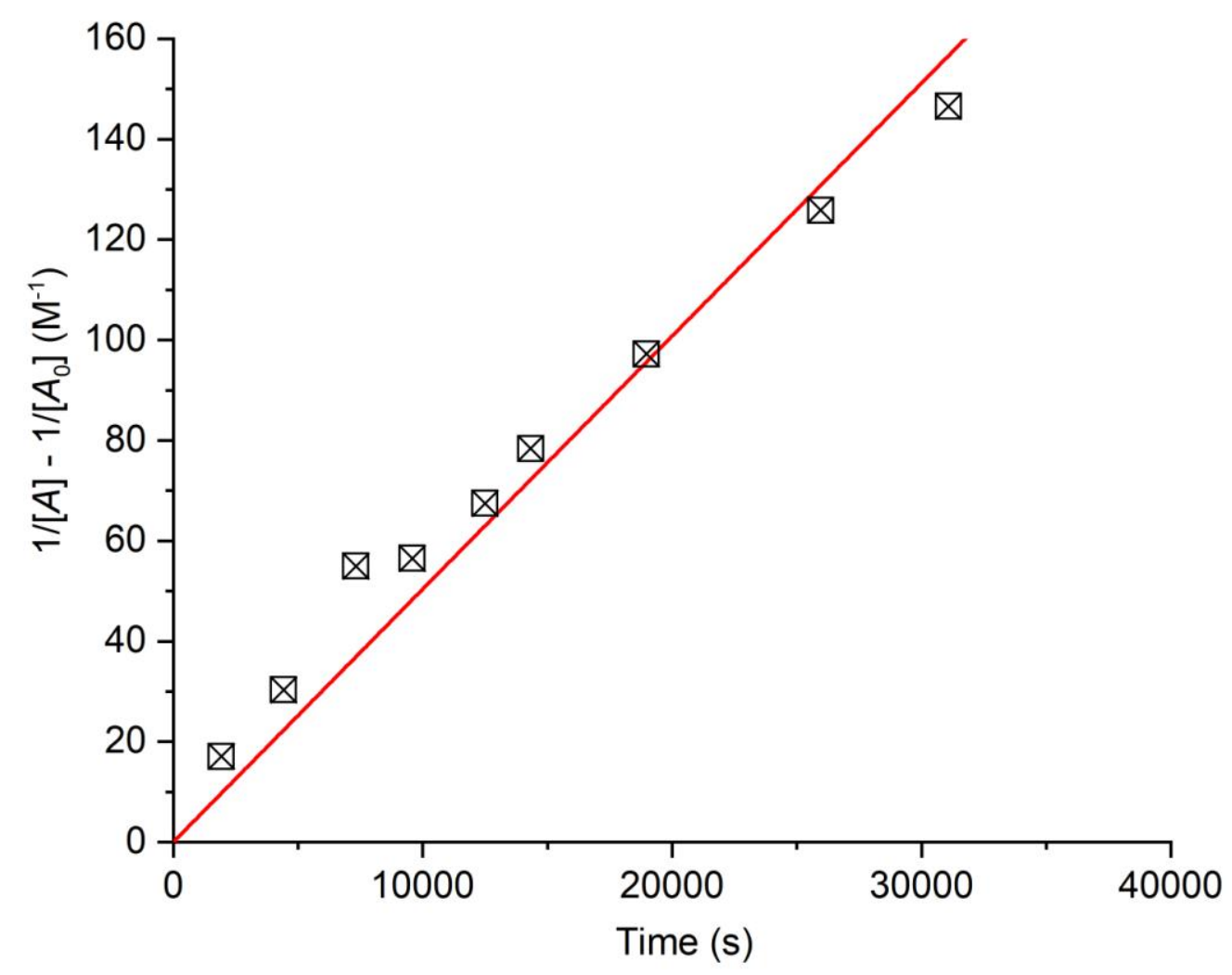

Figure S1. Linear fit of the change of the intensity of the ${ }^{19} \mathrm{~F}$ NMR signal of $\mathrm{F}_{3} \mathrm{C}-\mathrm{C} \equiv \mathrm{C}$ COOEt 3 at $-52.09 \mathrm{ppm}$ upon reaction with azido complex $\mathbf{2}$ to the second order rate law (see main text for details). 


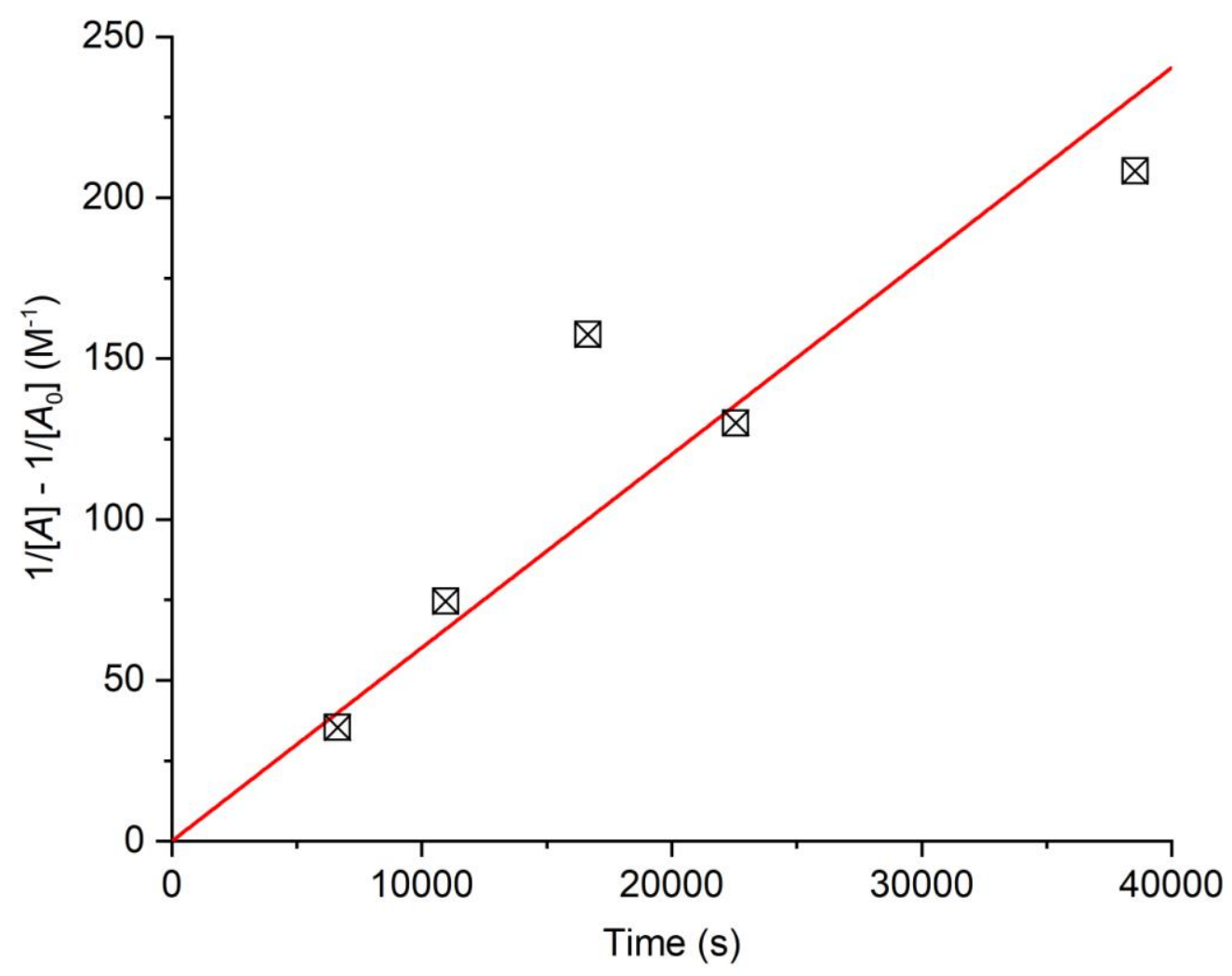

Figure S2. Linear fit of the change of the intensity of the ${ }^{31} \mathrm{P}$ NMR signal of trans[Pt(bodipy)( $\left.\left.\mathrm{N}_{3}\right)\left(\mathrm{PEt}_{3}\right)_{2}\right] \mathbf{2}$ at $13.45 \mathrm{ppm}$ upon reaction with alkyne $\mathbf{3}$ to the second order rate law (see main text for details). 


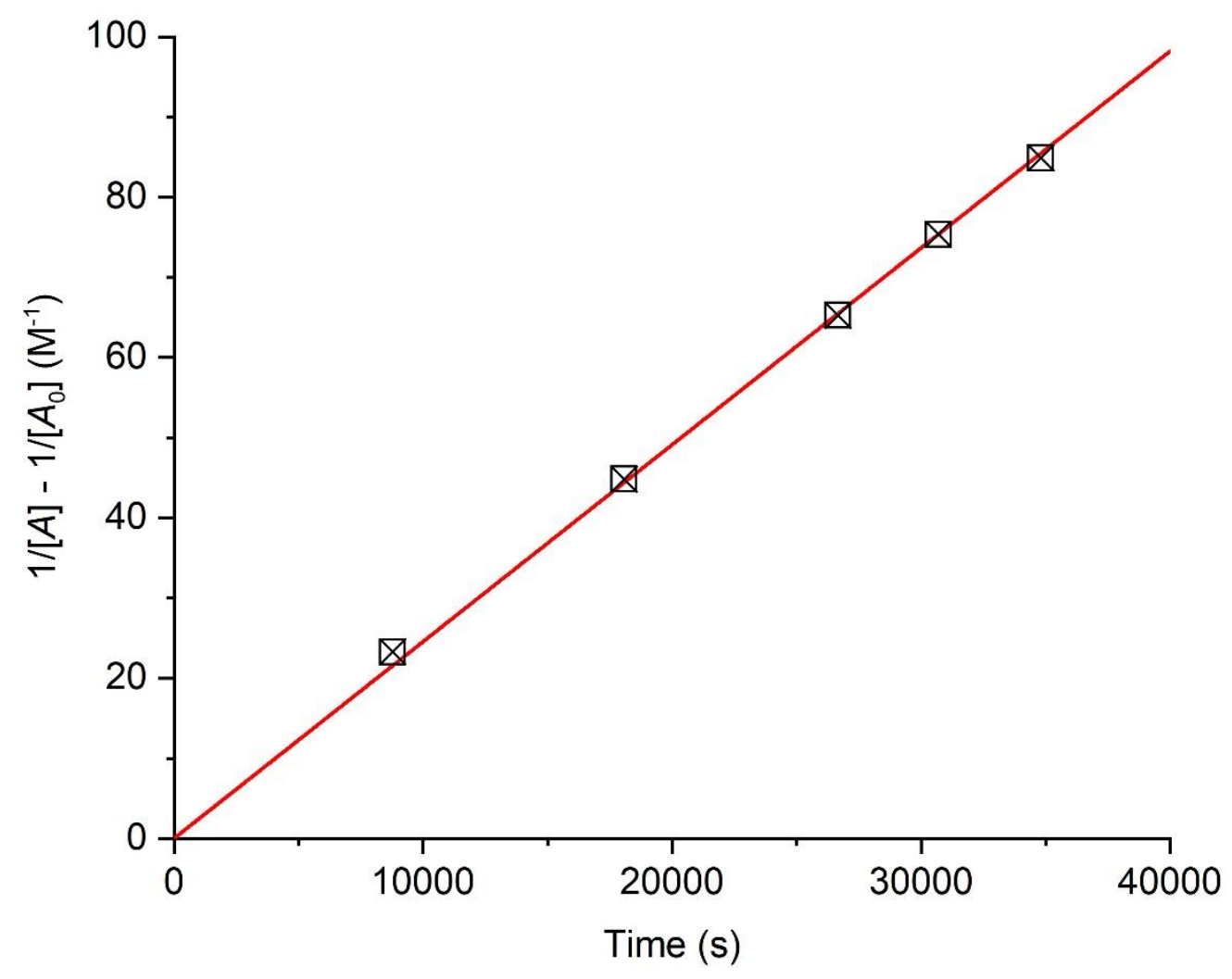

Figure S3. Linear fit of the change of the intensity of the ${ }^{1} \mathrm{H}$ NMR signal of the methyl ester proton signal of DMAD 4 at 3.85 ppm upon reaction with azido complex 2 to the second order rate law (see main text for details). 


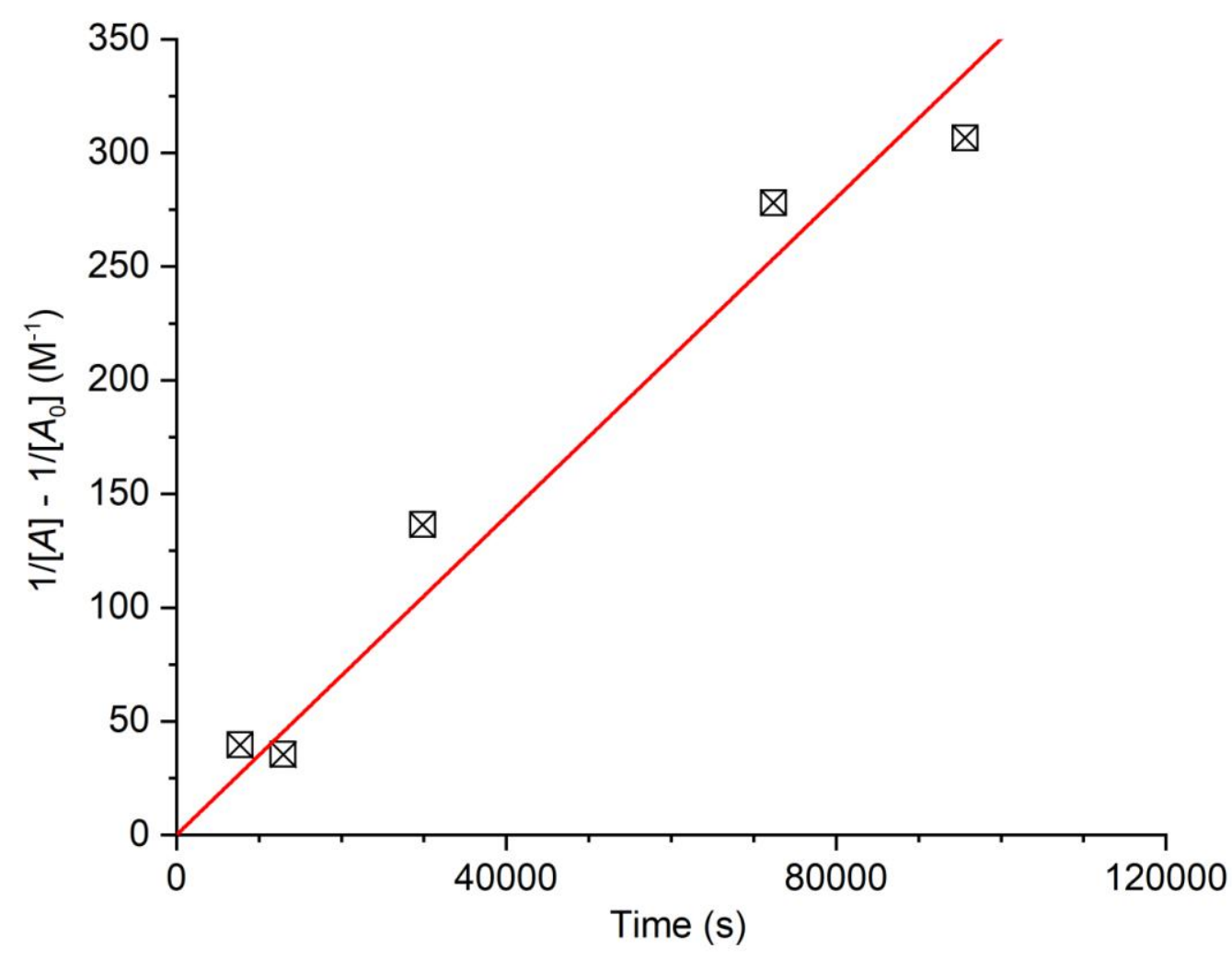

Figure S4. Linear fit of the change of the intensity of the ${ }^{31} \mathrm{P}$ NMR signal of trans$\left[\mathrm{Pt}(\right.$ bodipy $\left.)\left(\mathrm{N}_{3}\right)\left(\mathrm{PEt}_{3}\right)_{2}\right] 2$ at $13.45 \mathrm{ppm}$ upon reaction with DMAD 4 to the second order rate law (see main text for details). 
Section S2. Photophysical data
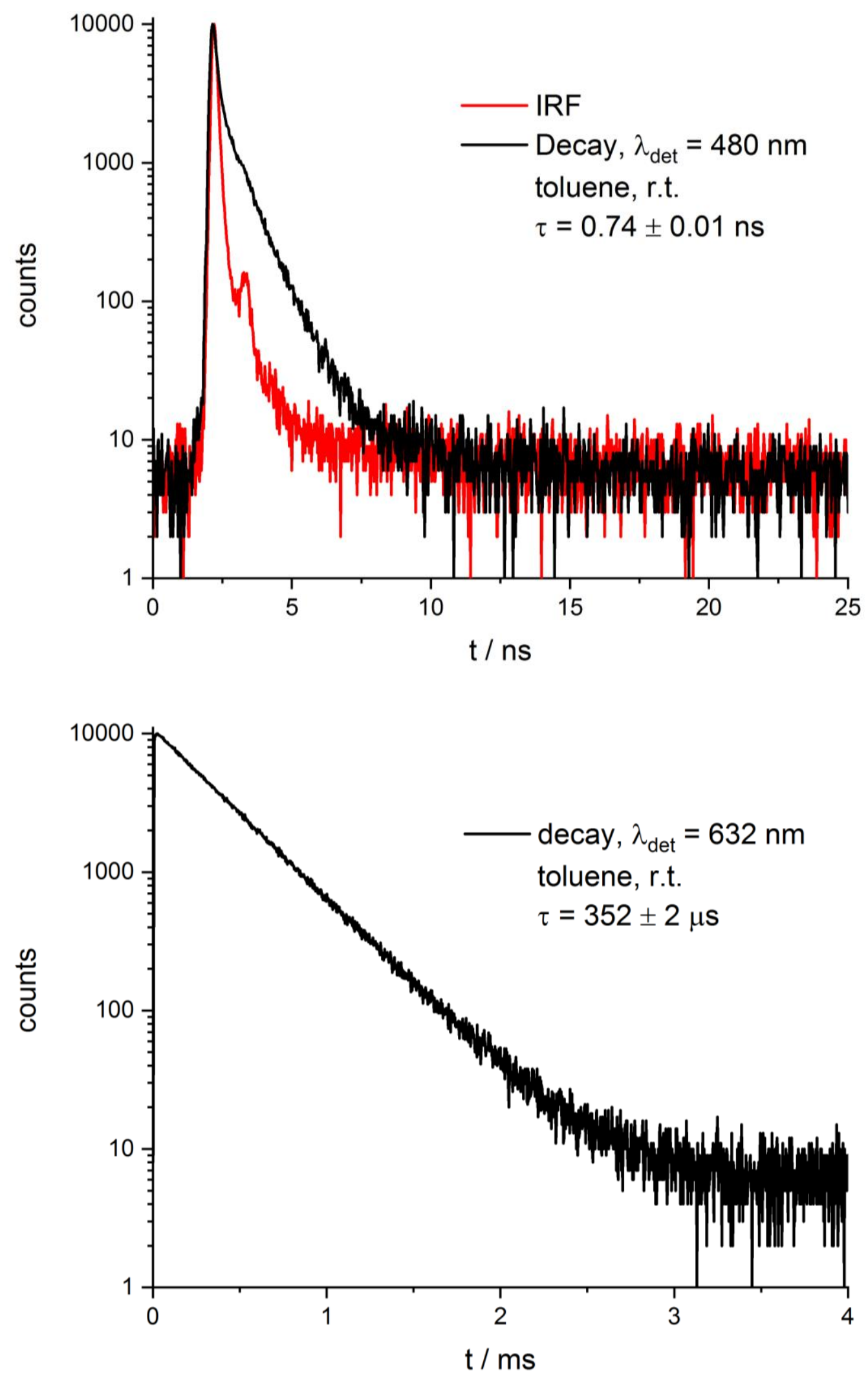

Figure S5. Lifetime decays of trans-[Pt(bodipy) $\left.\left(\mathrm{N}_{3}\right)\left(\mathrm{PEt}_{3}\right)_{2}\right] 2$ 

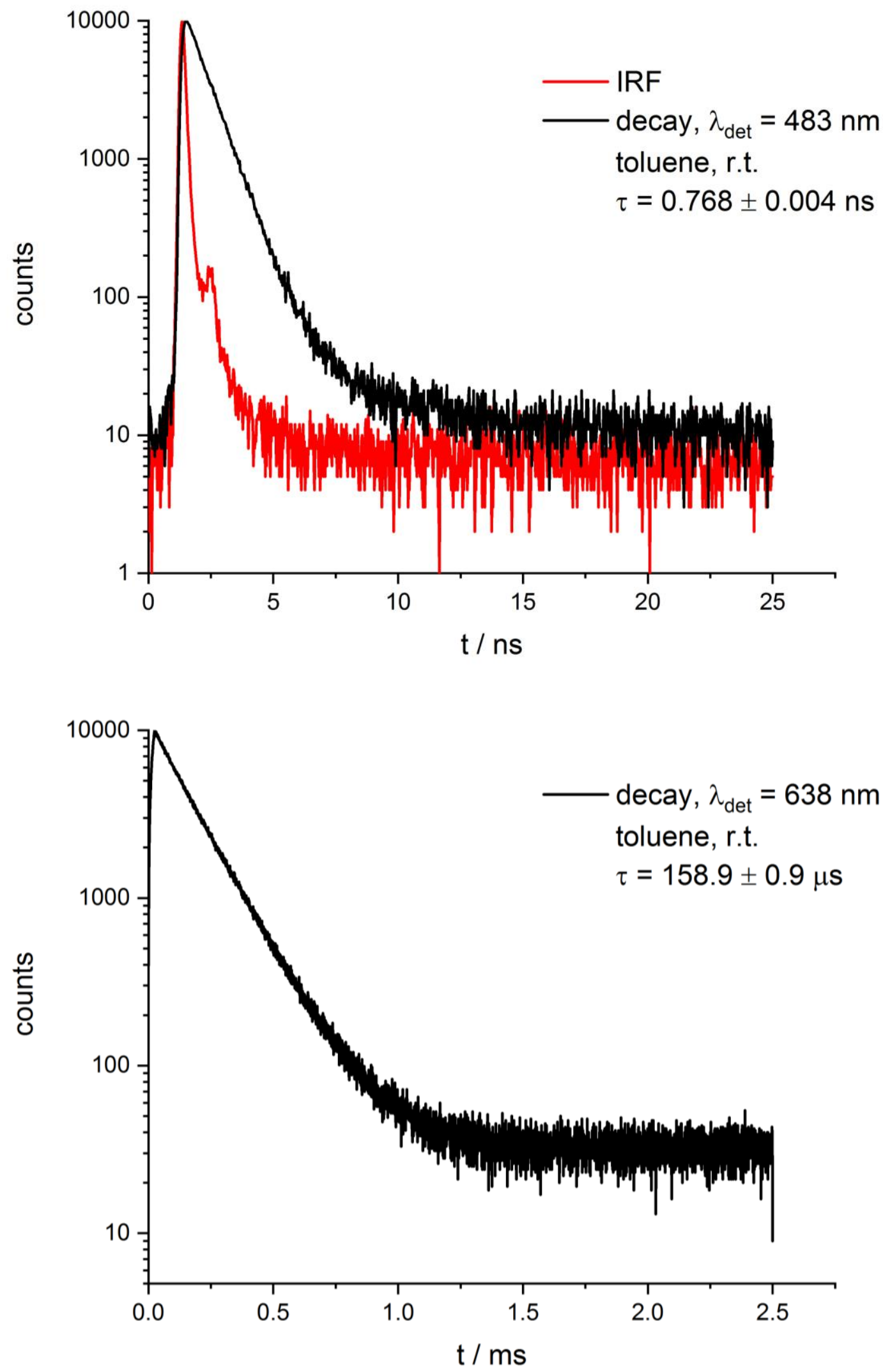

Figure S6. Lifetime decays of $\left[\mathrm{Pt}(\right.$ bodipy $)\left(\right.$ triazolate $\left.\left.{ }^{\mathrm{CF} 3, \mathrm{COOEt}}\right)\left(\mathrm{PEt}_{3}\right)_{2}\right] \mathbf{5}$ 

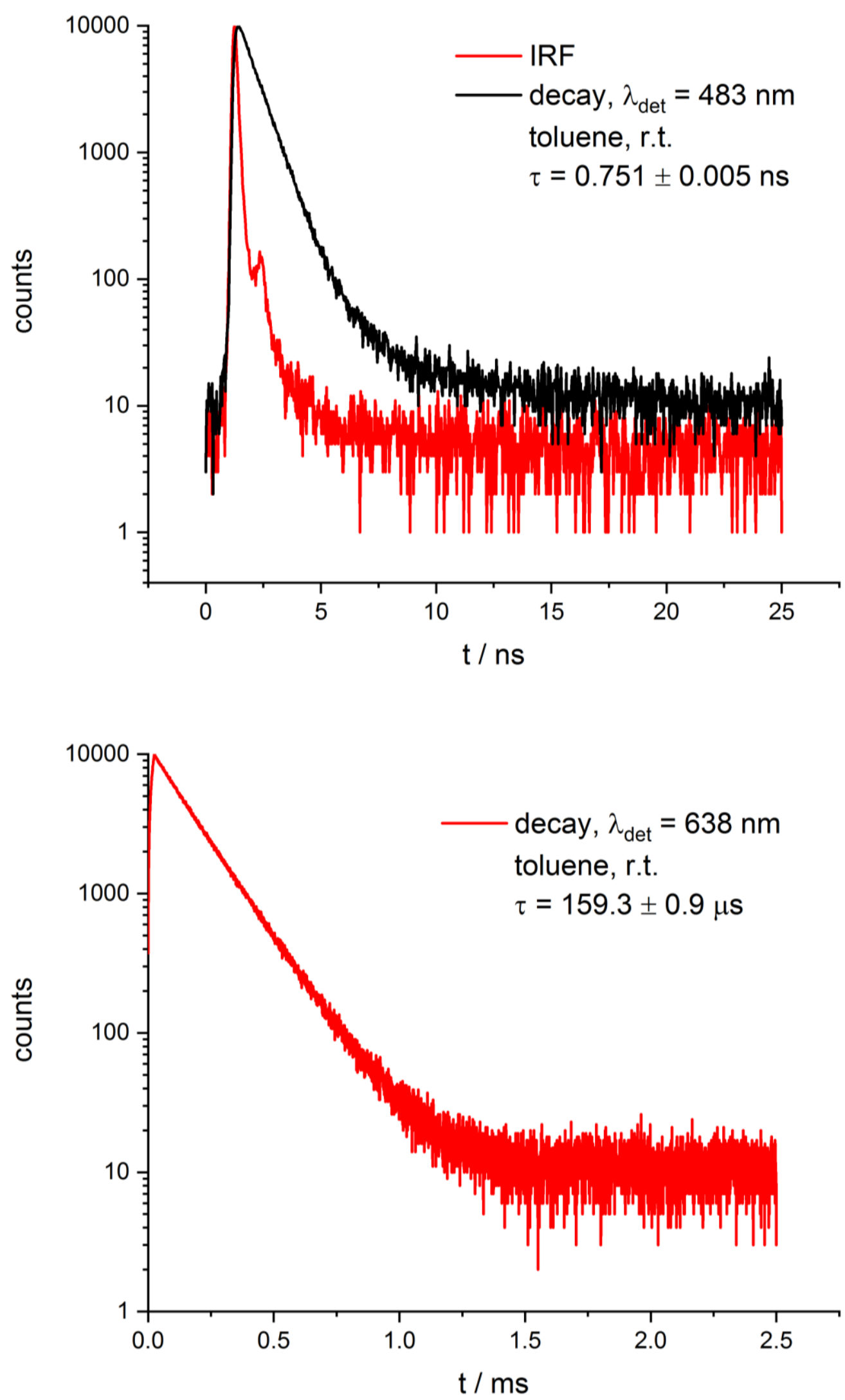

Figure S7. Lifetime decays of $\left[\mathrm{Pt}(\right.$ bodipy $)\left(\right.$ triazolate $\left.\left.^{\mathrm{COOCH} 3, \mathrm{COOCH} 3}\right)\left(\mathrm{PEt}_{3}\right)_{2}\right] 6$ 
Section S3. DFT calculations

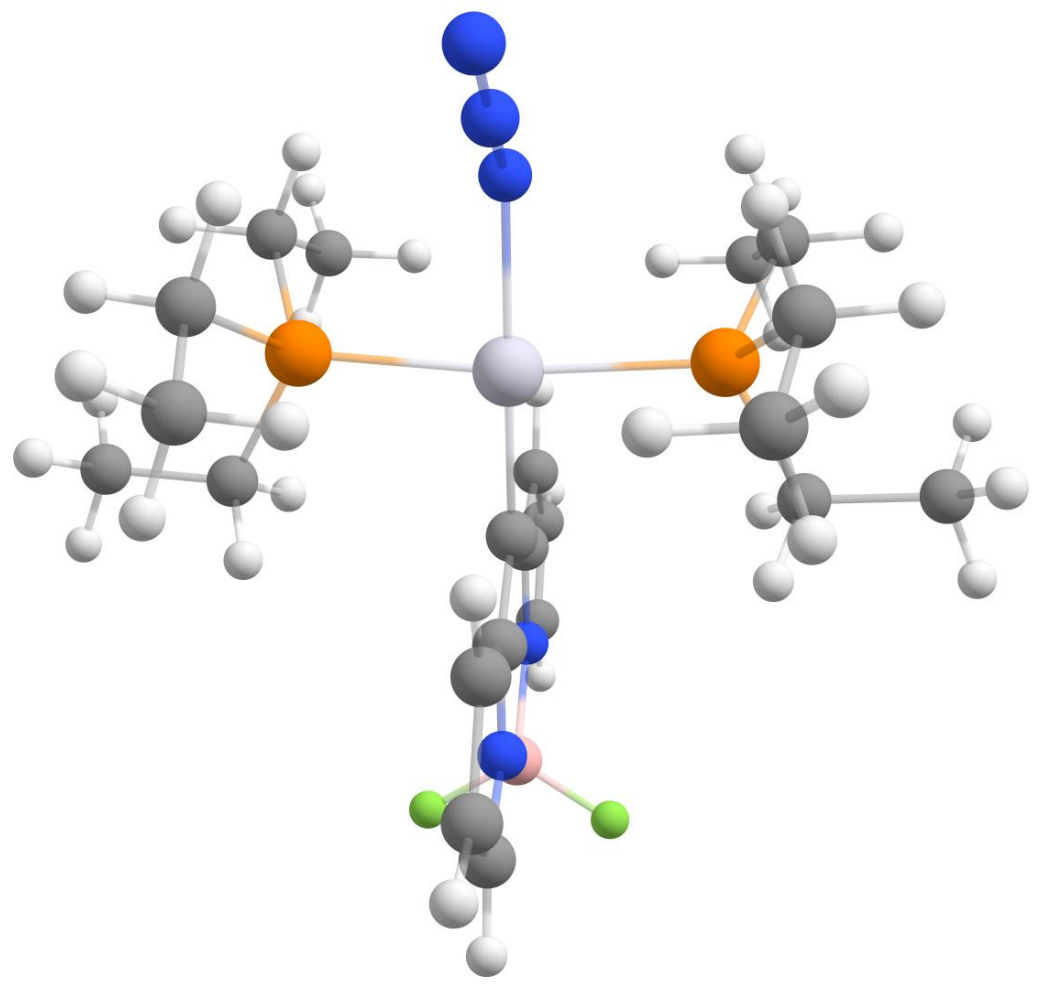

Figure S8. Optimized structure of trans-[Pt(bodipy)( $\left.\left.\mathrm{N}_{3}\right)\left(\mathrm{PEt}_{3}\right)_{2}\right] 2$

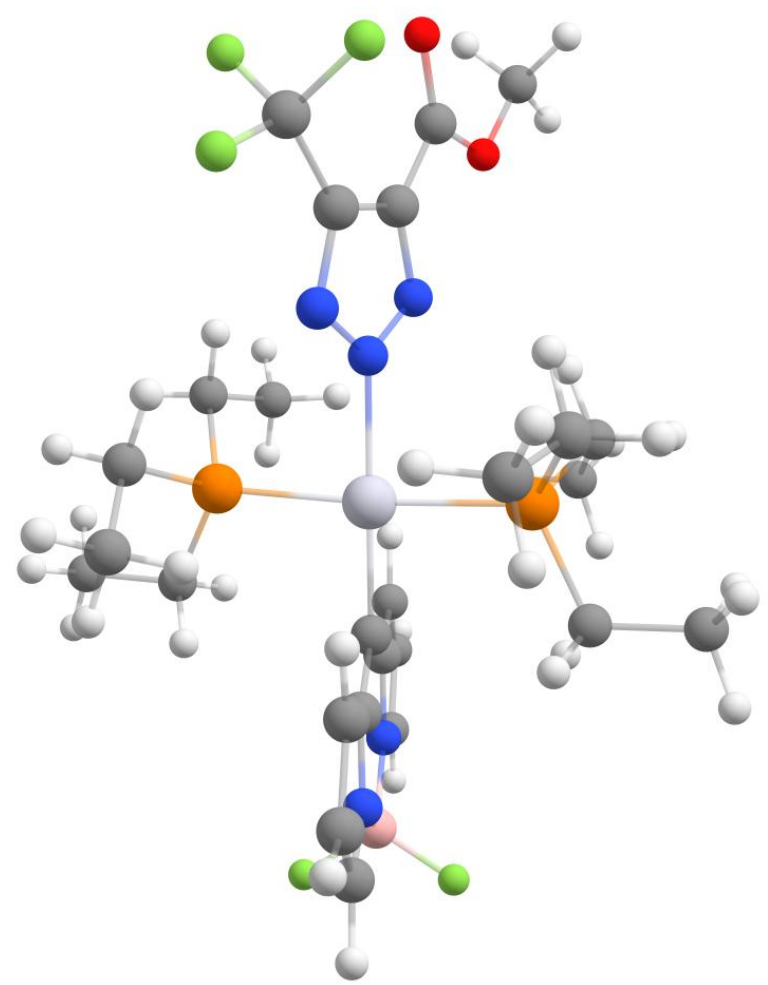

Figure S9. Optimized structure of trans-[Pt(bodipy)(triazolate $\left.\left.{ }^{\mathrm{CF} 3, \mathrm{COOE} t}\right)\left(\mathrm{PEt}_{3}\right)_{2}\right] 5$ 


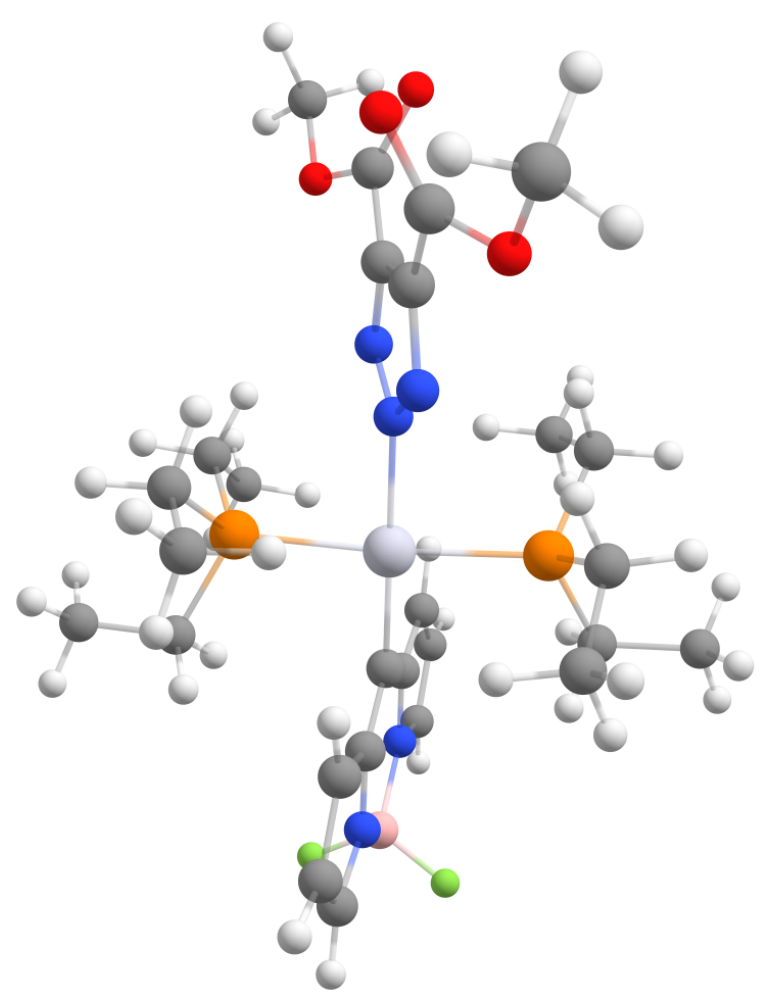

Figure S10. Optimized structure of trans-[Pt(bodipy) $\left(\right.$ triazolate $\left.\left.^{\mathrm{COOCH} 3, \mathrm{COOCH} 3}\right)\left(\mathrm{PEt}_{3}\right)_{2}\right] 6$

Table S1. Comparison of experimental and calculated azido and ester carbonyl group vibrational frequencies $\left(\right.$ in $\left.\mathrm{cm}^{-1}\right)$.

\begin{tabular}{|c|c|c|c|}
\hline Compound & & $v\left(\mathbf{N}_{3}\right)$ & $v(\mathrm{C}=\mathrm{O})$ \\
\hline \multirow{3}{*}{2} & $\exp$ & 2055 & \\
\hline & calc & 2075 & \\
\hline & diff. calc-exp & +20 & \\
\hline \multirow{3}{*}{5} & $\exp$ & & 1728 \\
\hline & calc & & 1693 \\
\hline & diff. calc-exp & & -35 \\
\hline \multirow{3}{*}{6} & $\exp$ & & 1725 \\
\hline & calc & & 1707,1689 \\
\hline & diff. calc-exp & & $-18,-36$ \\
\hline
\end{tabular}




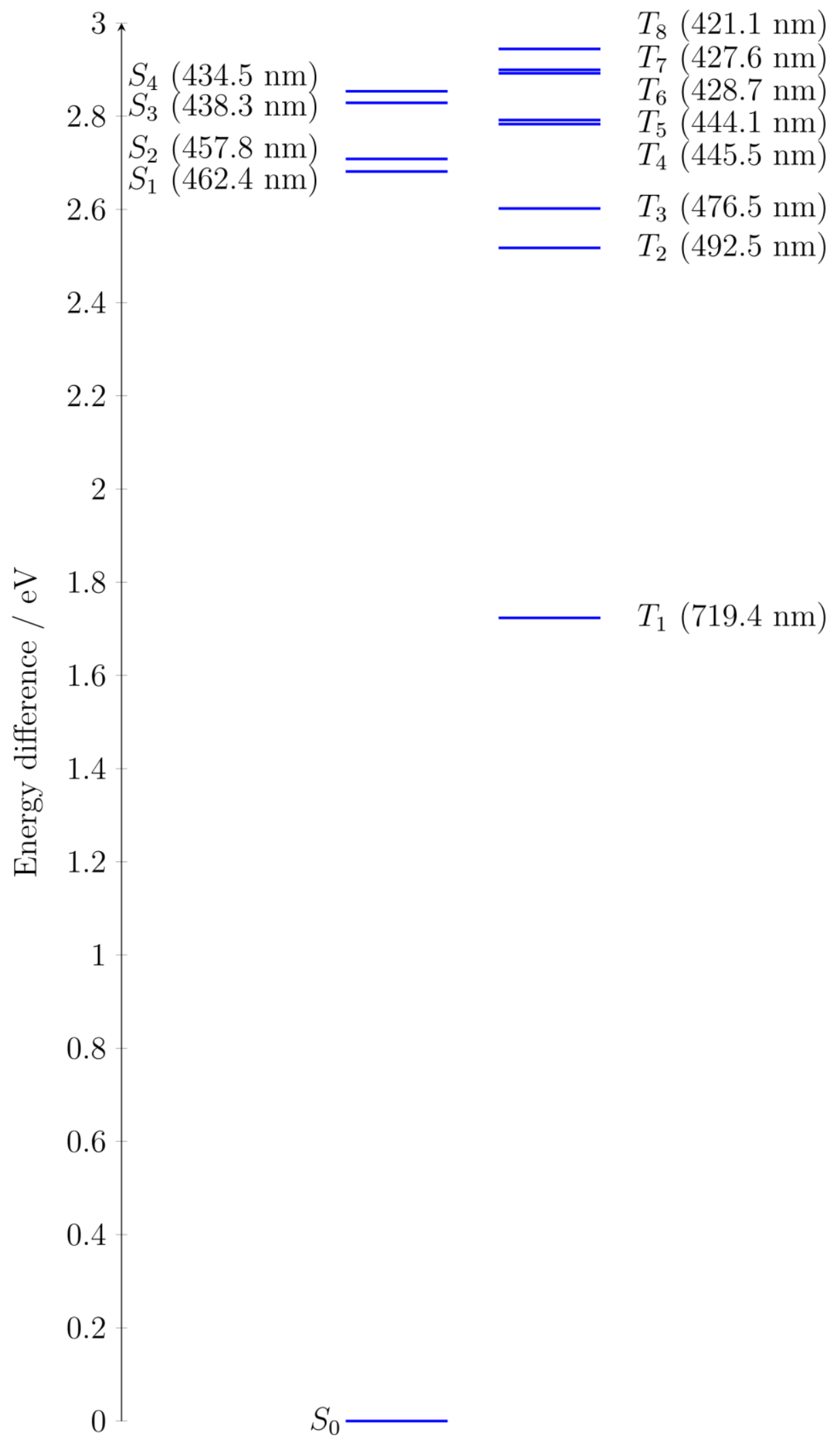

Figure S11. Energy level diagramm for trans-[Pt(bodipy)( $\left.\left.\mathrm{N}_{3}\right)\left(\mathrm{PEt}_{3}\right)_{2}\right] 2$ 


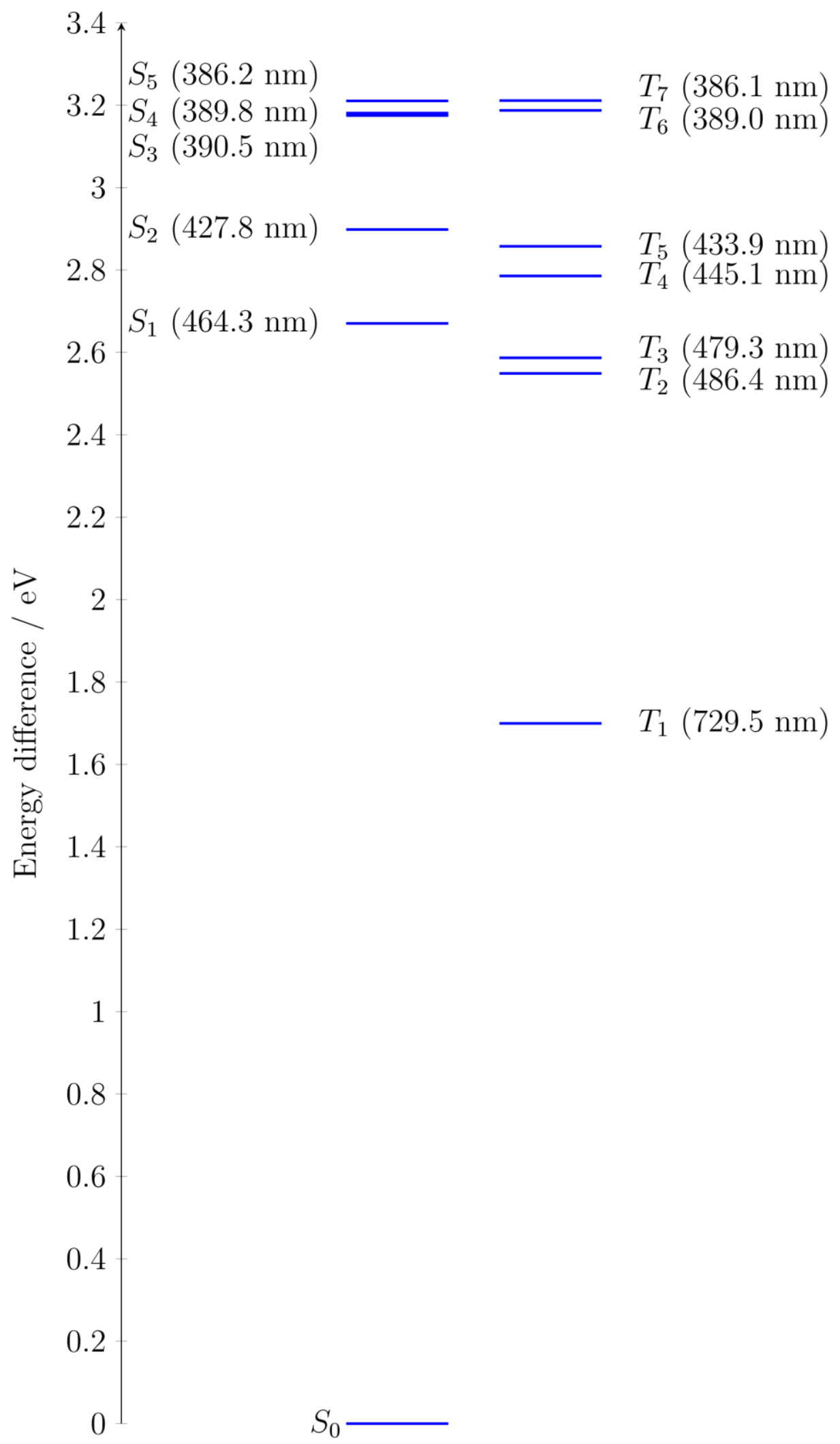

Figure S12. Energy level diagramm for trans-[Pt(bodipy)(triazolate $\left.\left.{ }^{\mathrm{CF} 3, \mathrm{COOEt}}\right)\left(\mathrm{PEt}_{3}\right)_{2}\right] \mathbf{5}$ 


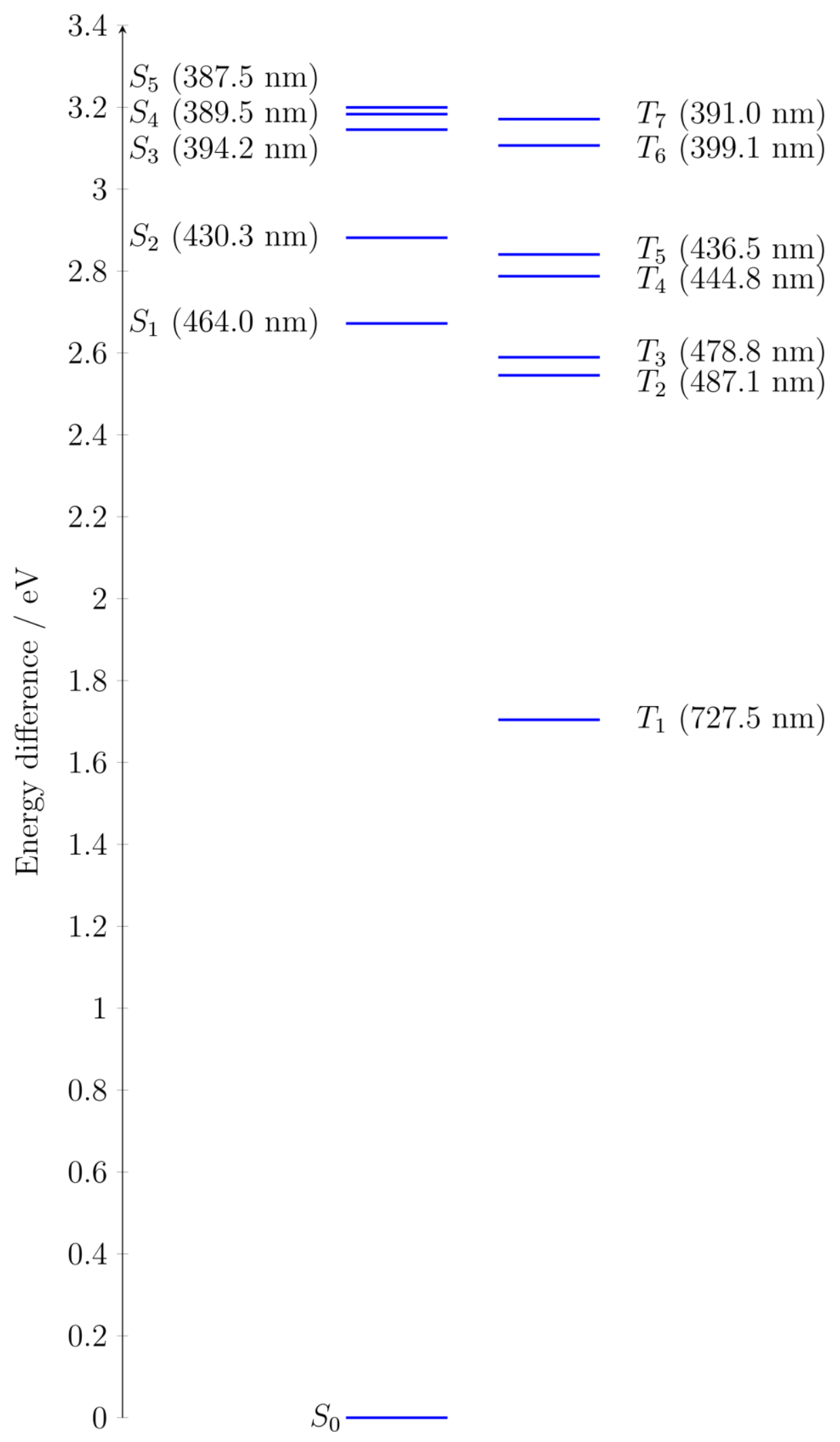

Figure S13. Energy level diagramm for trans-[Pt(bodipy)(triazolate $\left.\left.{ }^{\mathrm{COOCH} 3, \mathrm{COOCH} 3}\right)\left(\mathrm{PEt}_{3}\right)_{2}\right] 6$ 
Table S2. Relevant excited states and their main compositions for trans-[Pt(bodipy)( $\left.\left.\mathrm{N}_{3}\right)\left(\mathrm{PEt}_{3}\right)_{2}\right] 2$

\begin{tabular}{|c|c|c|c|}
\hline State & $\lambda \mathrm{nm}$ & Type of transition & Difference density plot \\
\hline $\mathrm{T} 8$ & 421.1 & $\begin{array}{c}\text { LLCT azido } \rightarrow \text { BODIPY } \\
\text { ILCT azide }\end{array}$ & \\
\hline $\mathrm{T} 7$ & 427.6 & $\begin{array}{c}\text { MLCT } \mathrm{Pt} \rightarrow \text { BODIPY } \\
\text { ILCT azide }\end{array}$ & \\
\hline T6 & 428.7 & $\begin{array}{c}\text { MLCT } \mathrm{Pt} \rightarrow \text { BODIPY } \\
\text { ILCT azide }\end{array}$ & \\
\hline S4 & 434.5 & MLCT Pt $\rightarrow$ BODIPY & \\
\hline S3 & 438.3 & $\begin{array}{c}\text { MLCT } \mathrm{Pt} \rightarrow \text { BODIPY } \\
\text { LLCT azide } \rightarrow \text { BODIPY }\end{array}$ & \\
\hline
\end{tabular}




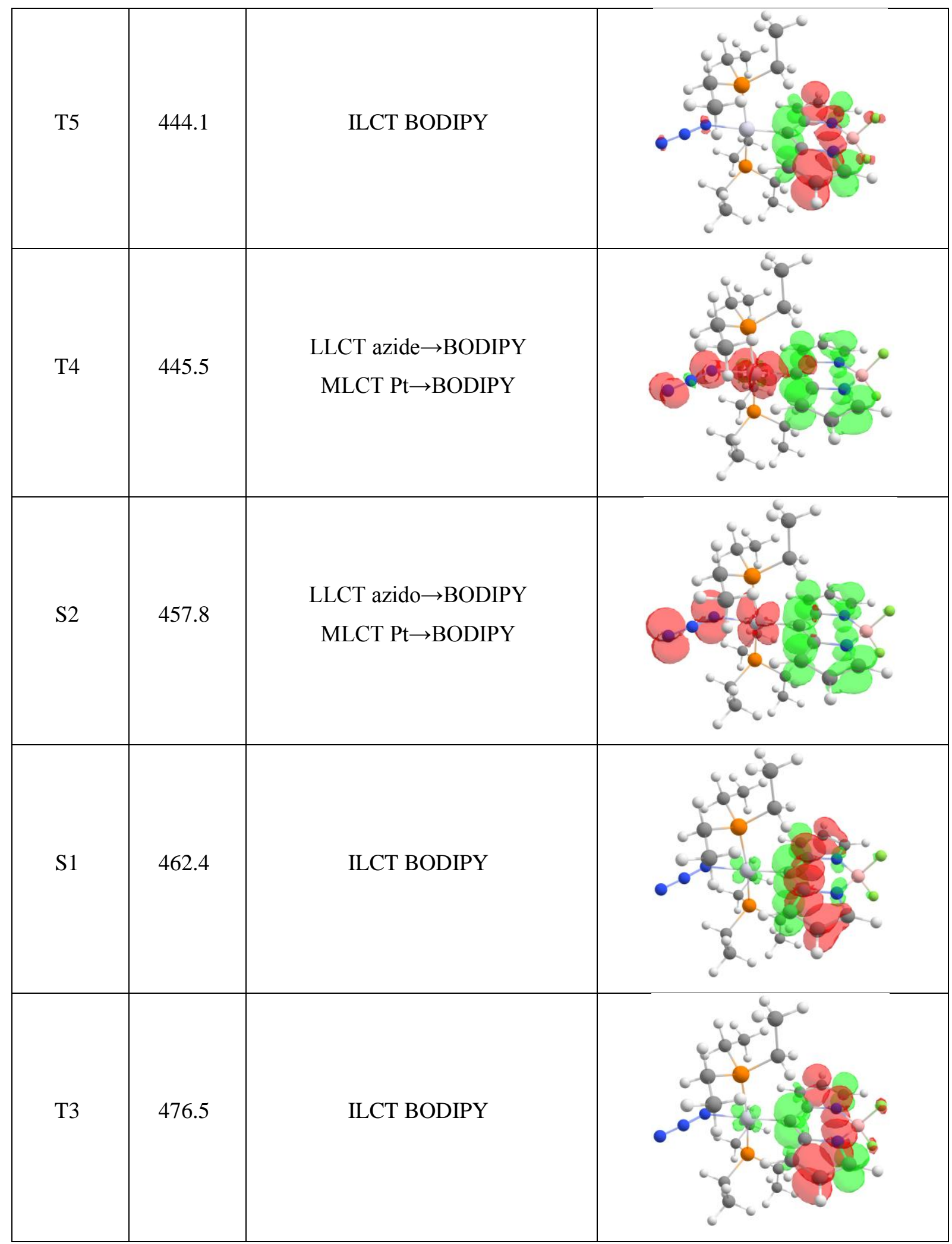



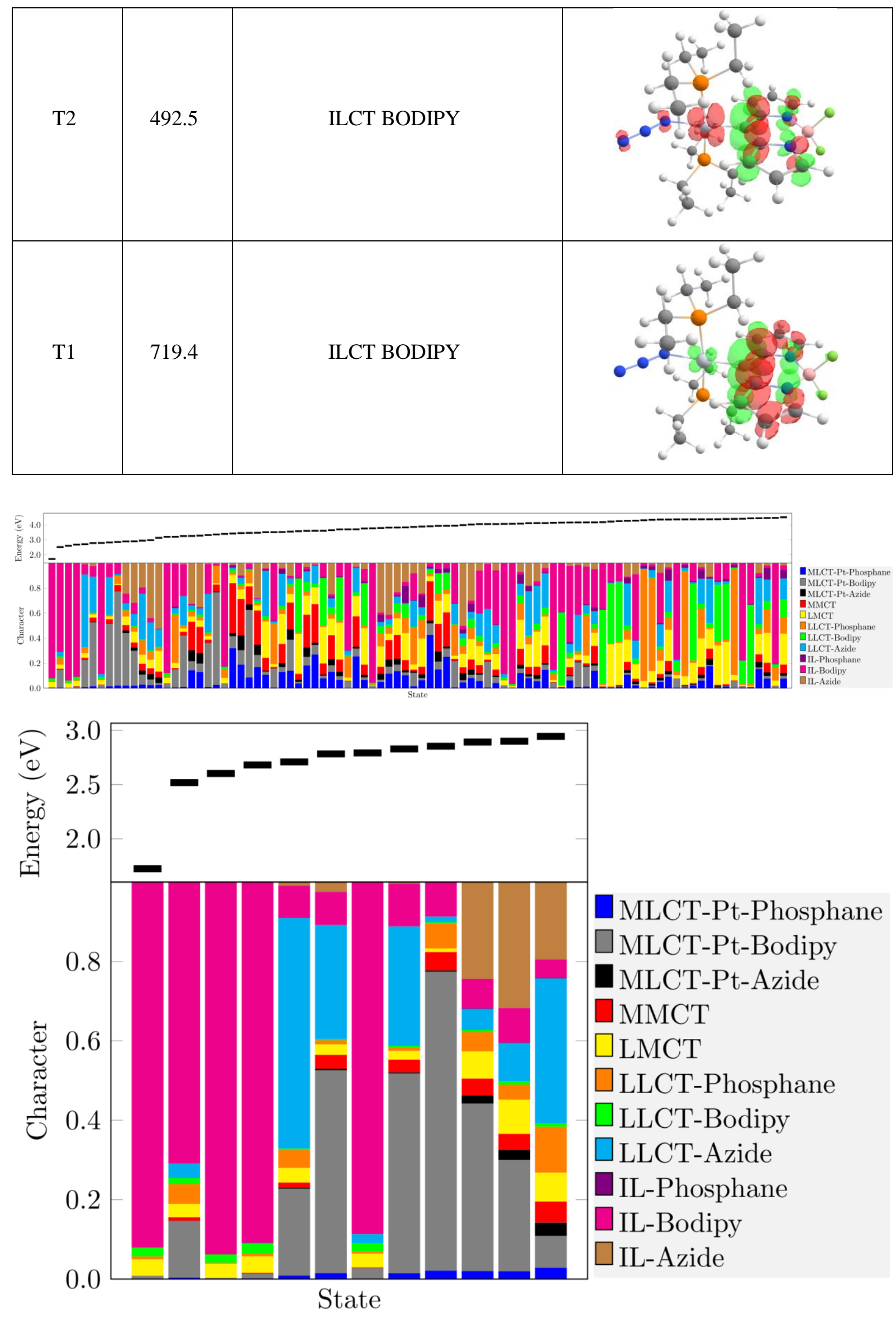
Table S3. Relevant excited states and their main compositions for trans$\left[\mathrm{Pt}\right.$ (bodipy)(triazolate $\left.\left.{ }^{\mathrm{CF} 3, \mathrm{COOEt}}\right)\left(\mathrm{PEt}_{3}\right)_{2}\right] 5$

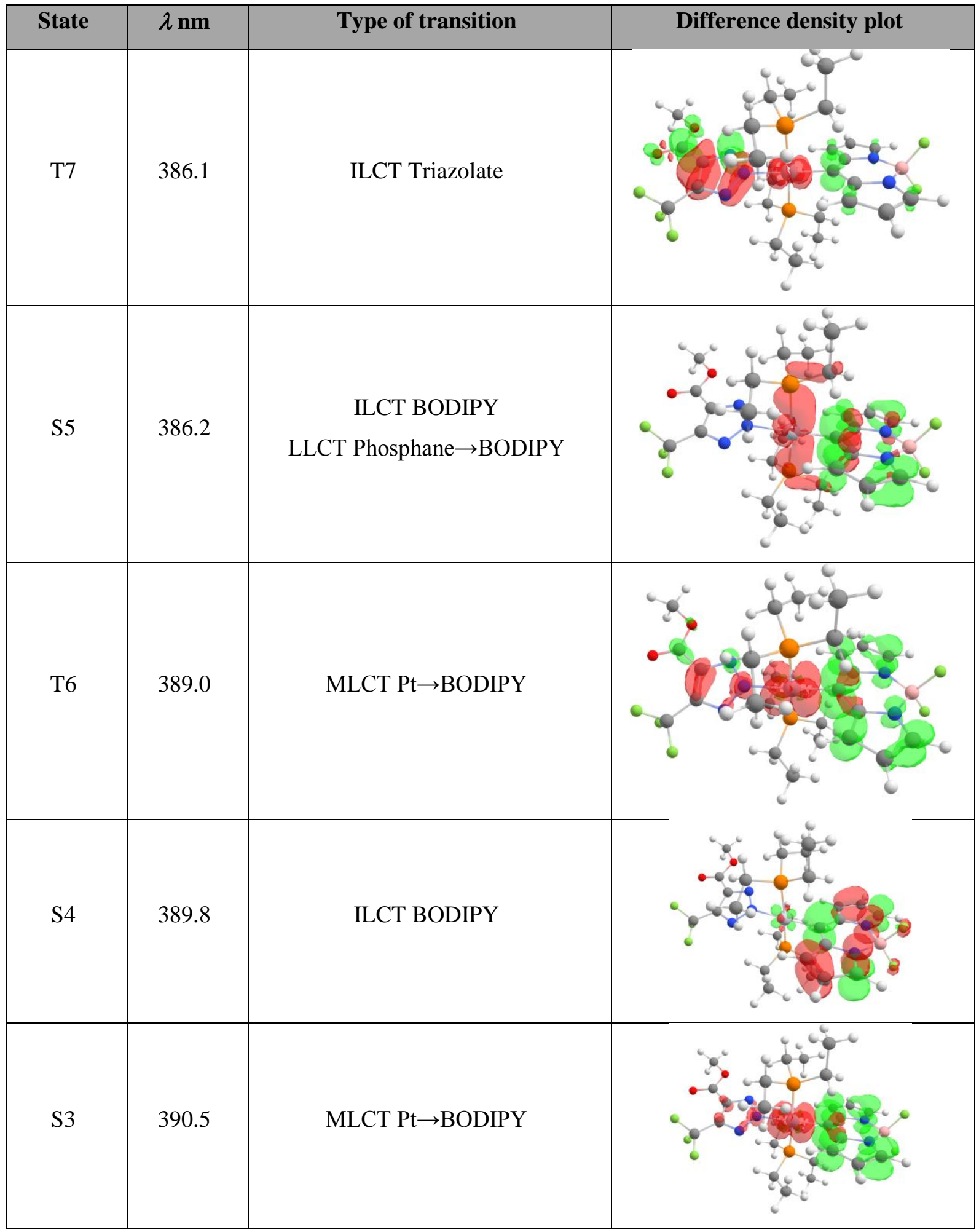




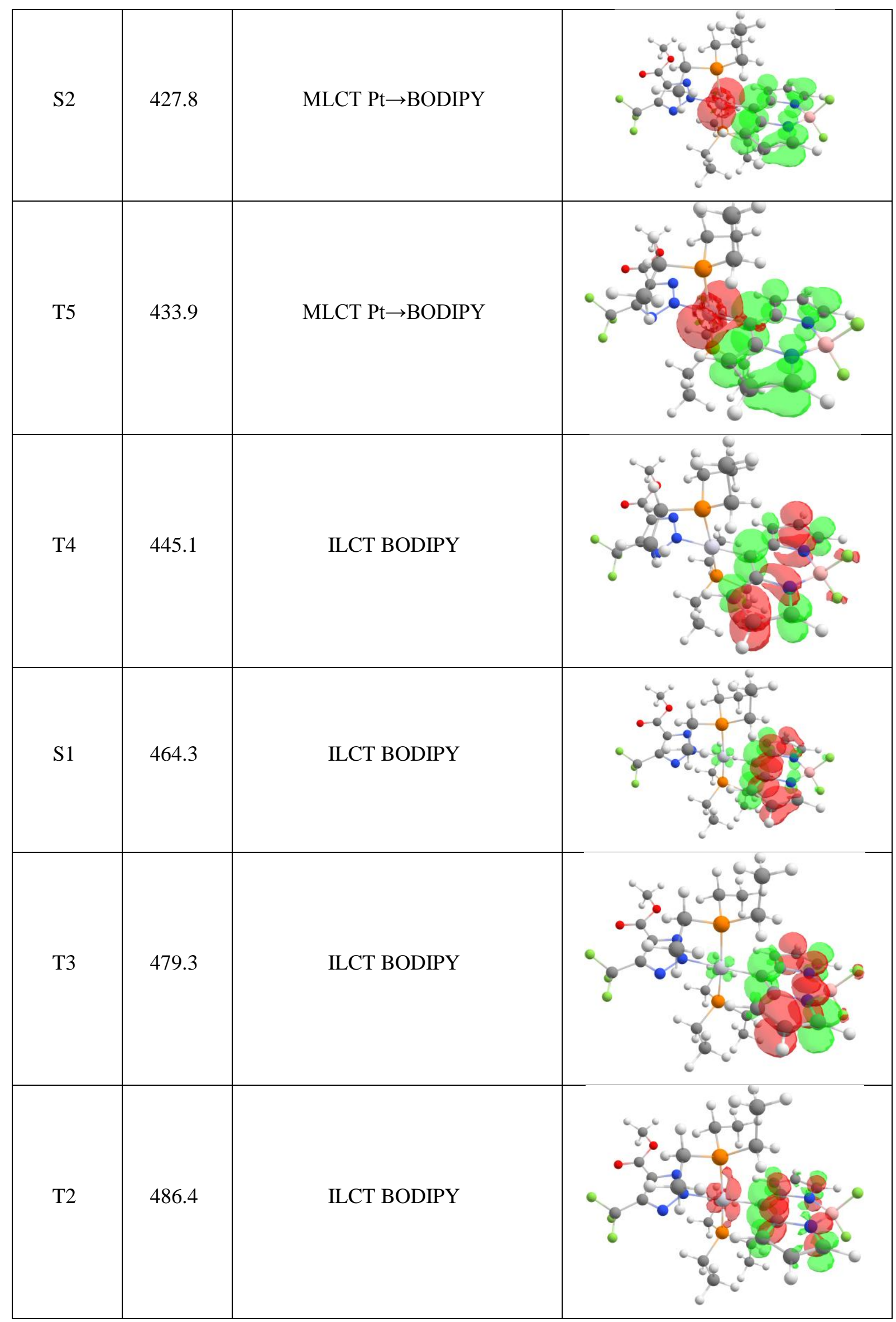



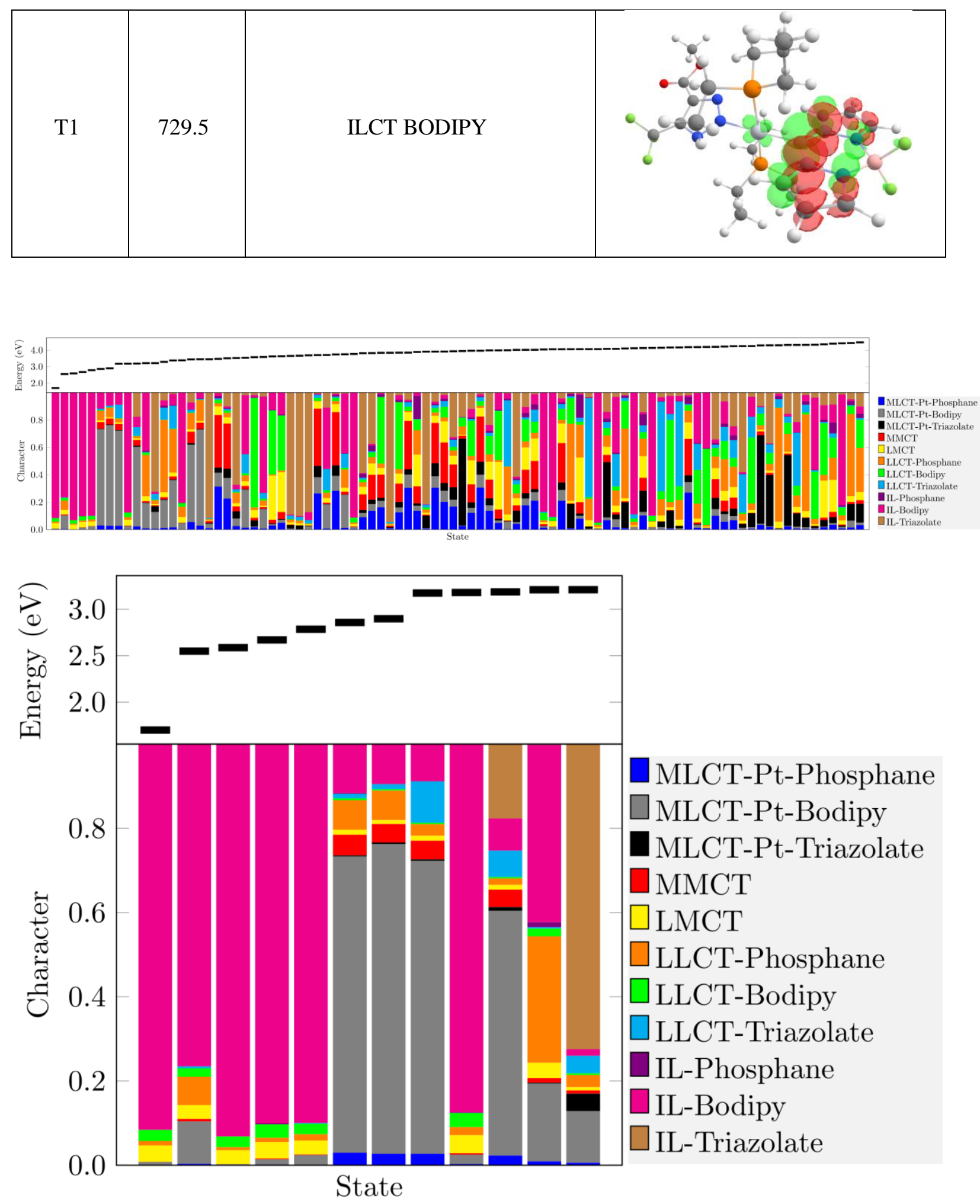
Table S4. Relevant excited states and their main compositions for trans$\left[\mathrm{Pt}\right.$ (bodipy)(triazolate $\left.\left.{ }^{\mathrm{COOCH} 3, \mathrm{COOCH}}\right)\left(\mathrm{PEt}_{3}\right)_{2}\right] 6$

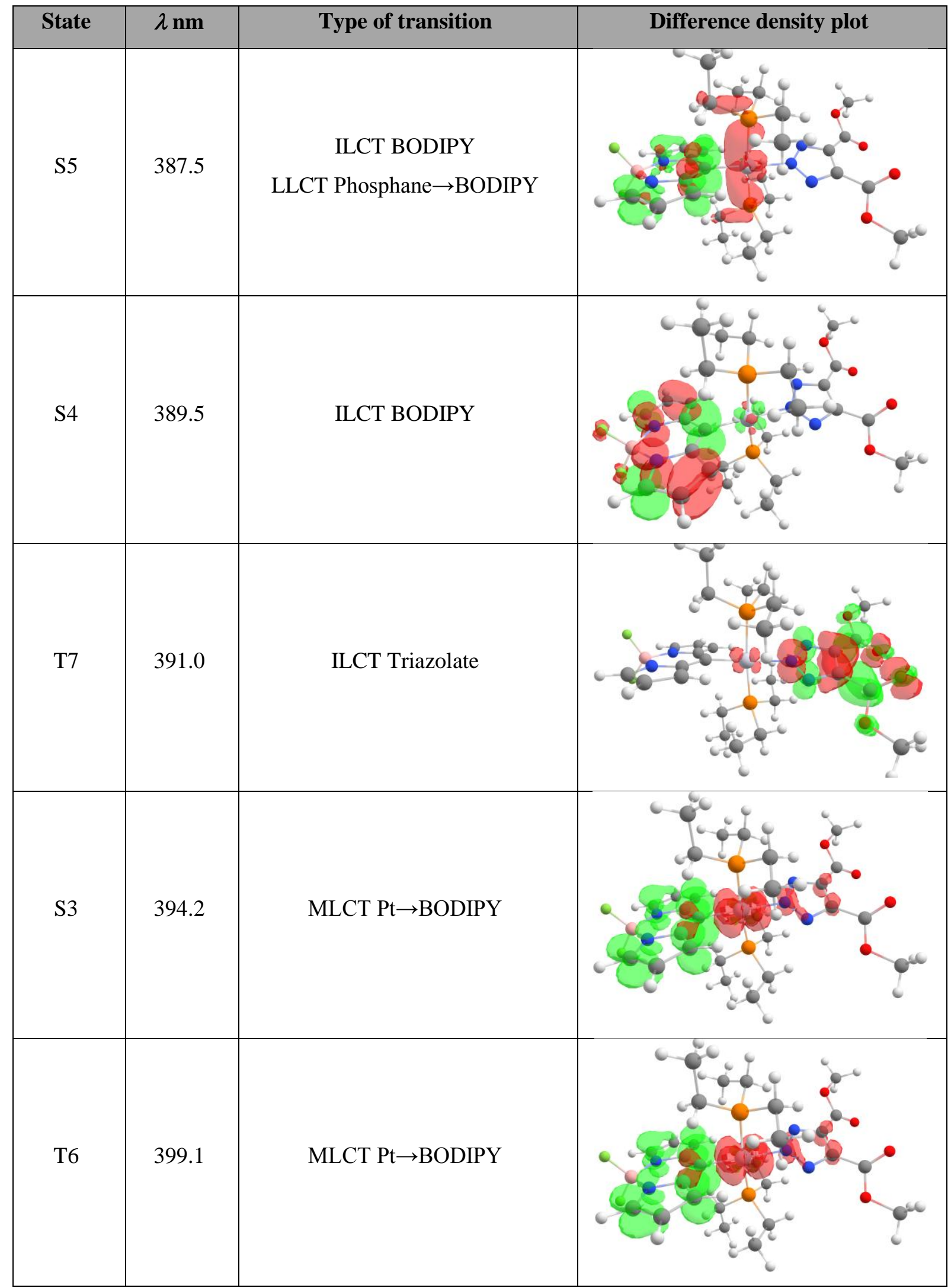




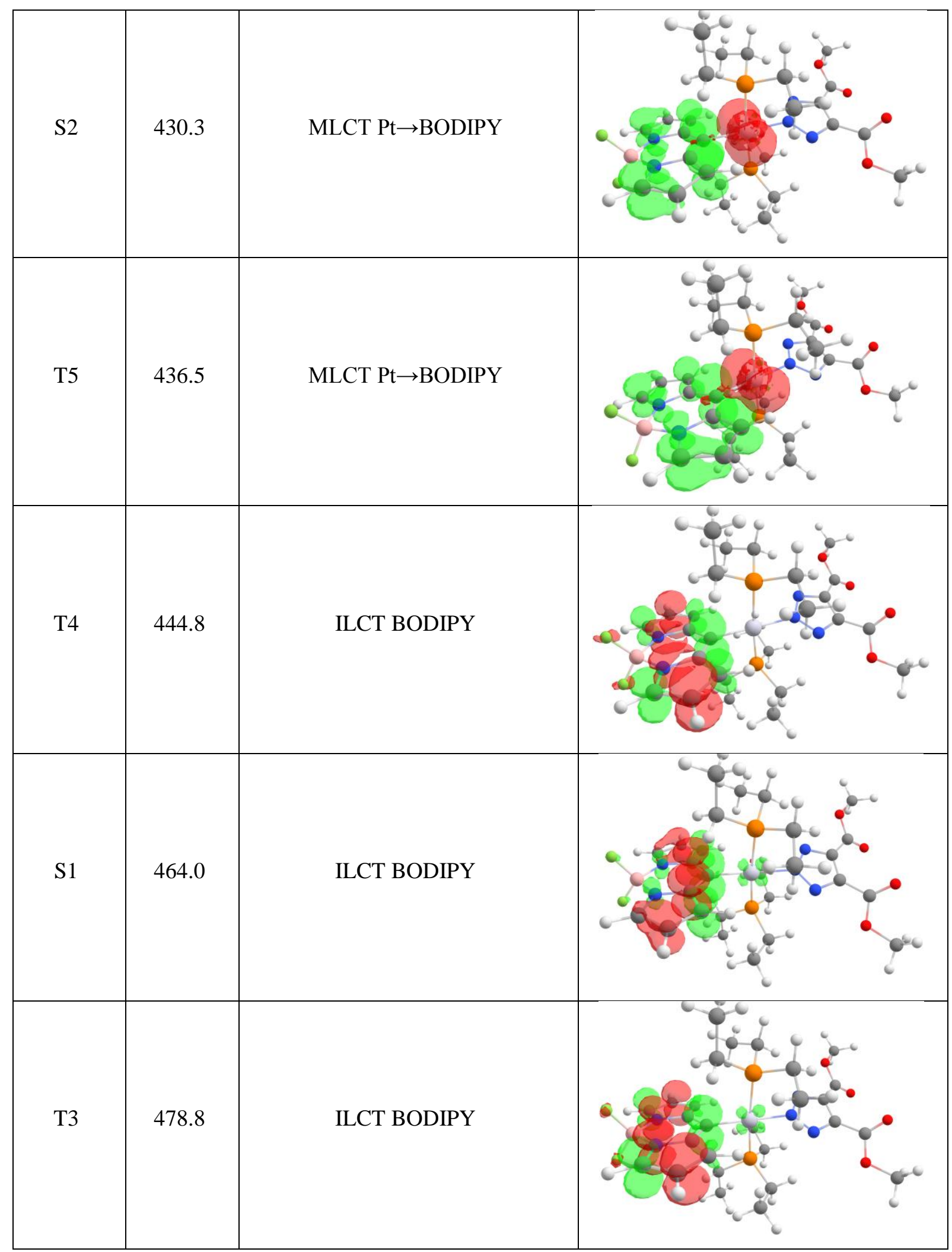



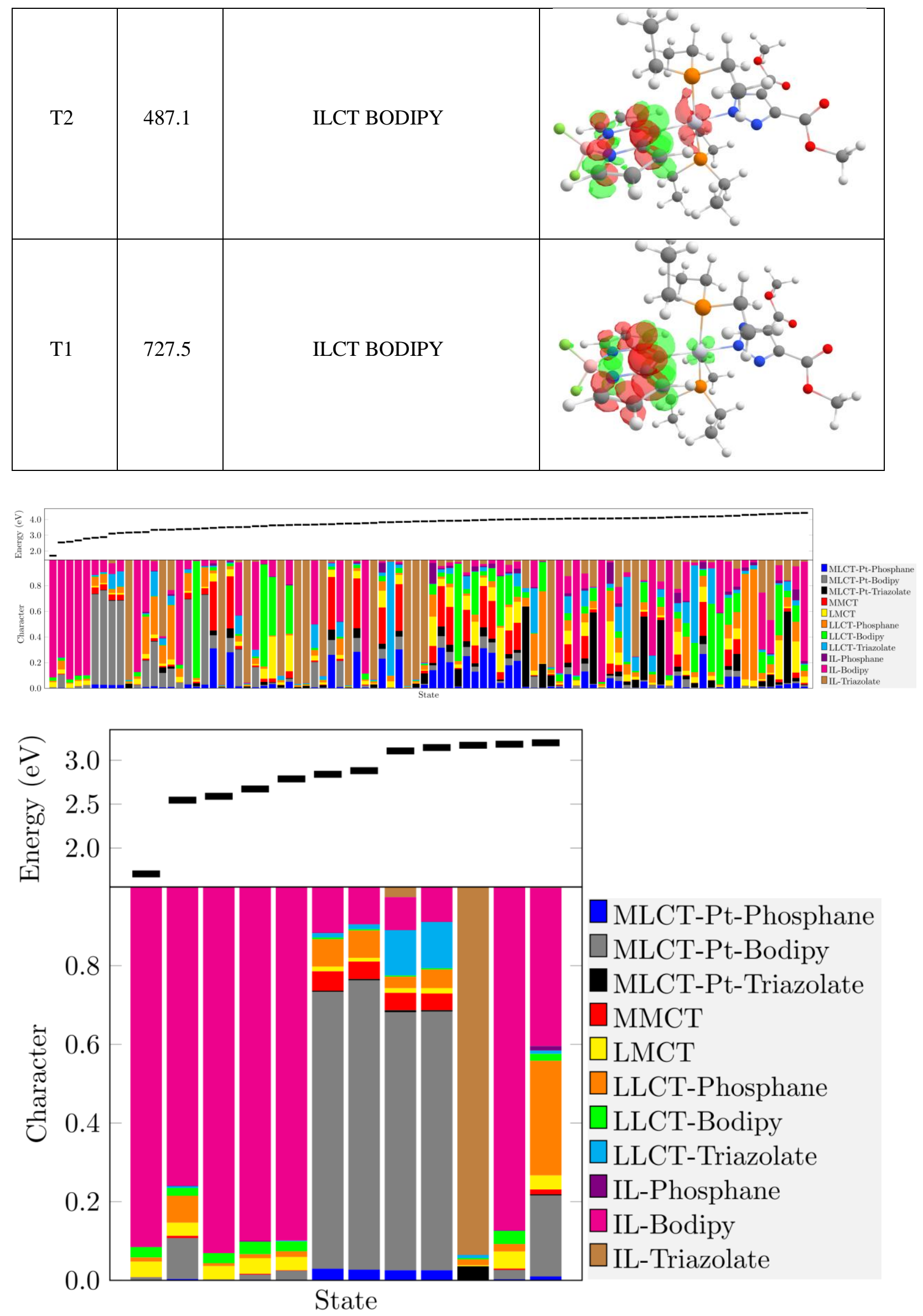
Section S4. Synthesis and characterization

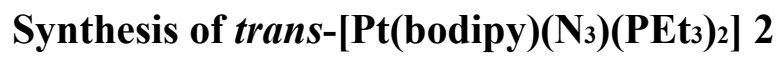<smiles></smiles>

a) AgOTf, $\mathrm{CD}_{2} \mathrm{Cl}_{2}$

b) $\mathrm{C}_{6} \mathrm{H}_{6} / \mathrm{CH}_{3} \mathrm{OH}, \mathrm{NaN}_{3}$<smiles></smiles>

Scheme S1. Synthesis of 2.

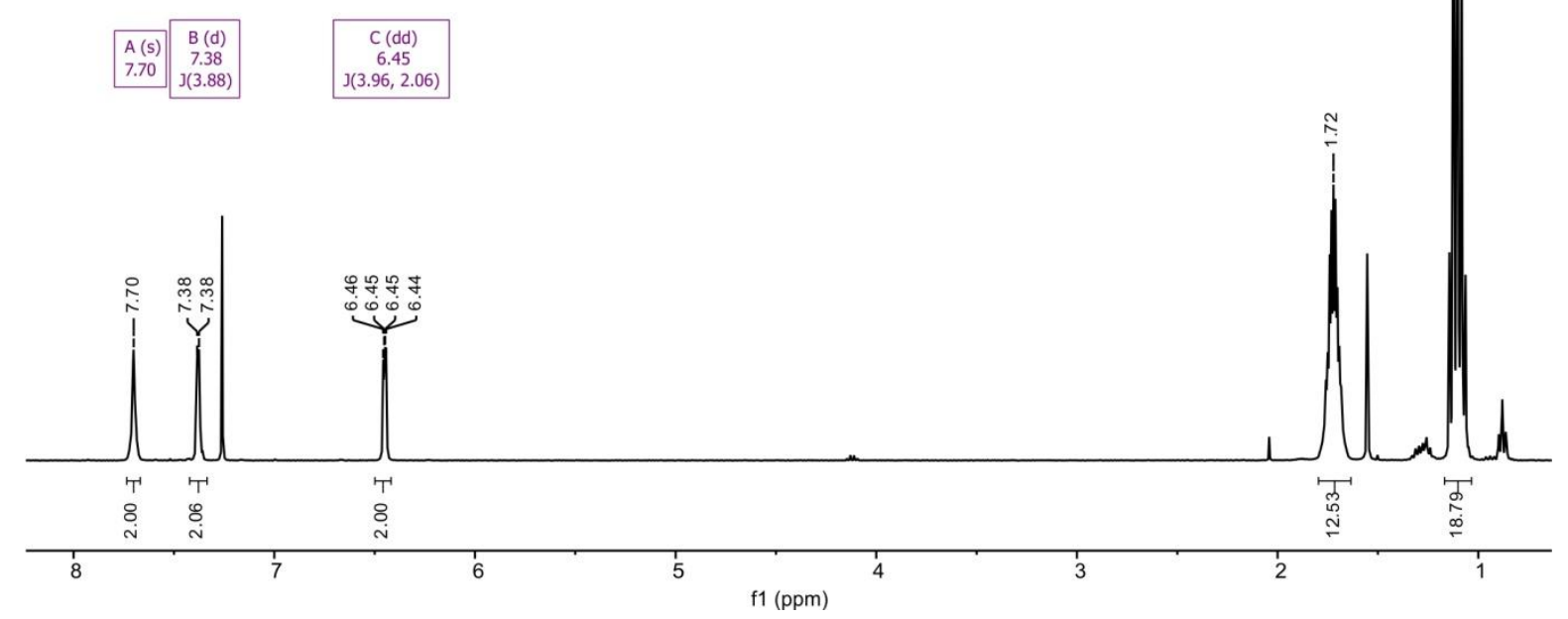

Figure S14. $400 \mathrm{MHz}{ }^{1} \mathrm{H} \mathrm{NMR}$ of 2 in $\mathrm{CDCl}_{3}$. 


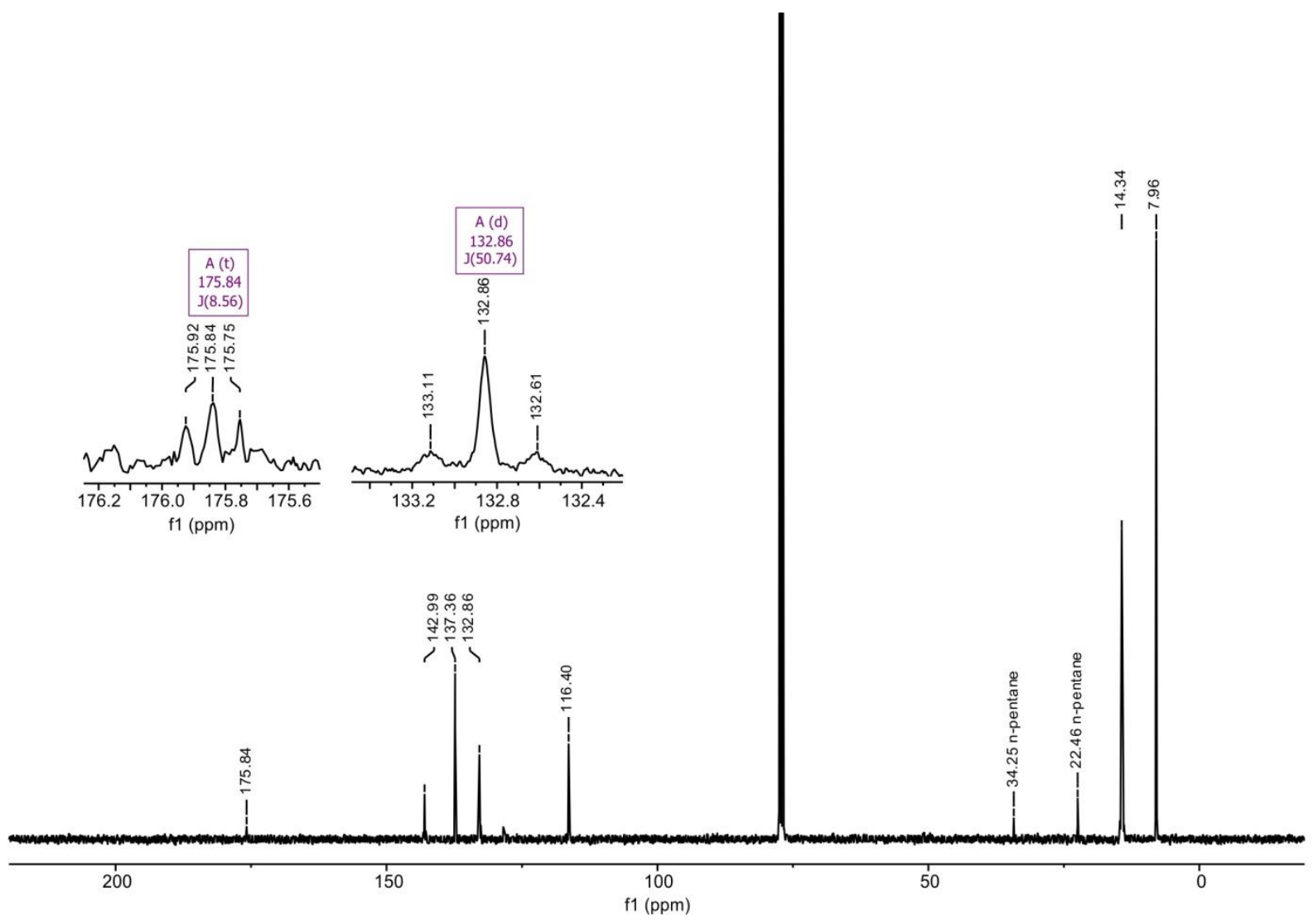

Figure S15. $100 \mathrm{MHz}{ }^{13} \mathrm{C}$ NMR of 2 in $\mathrm{CDCl}_{3}$.

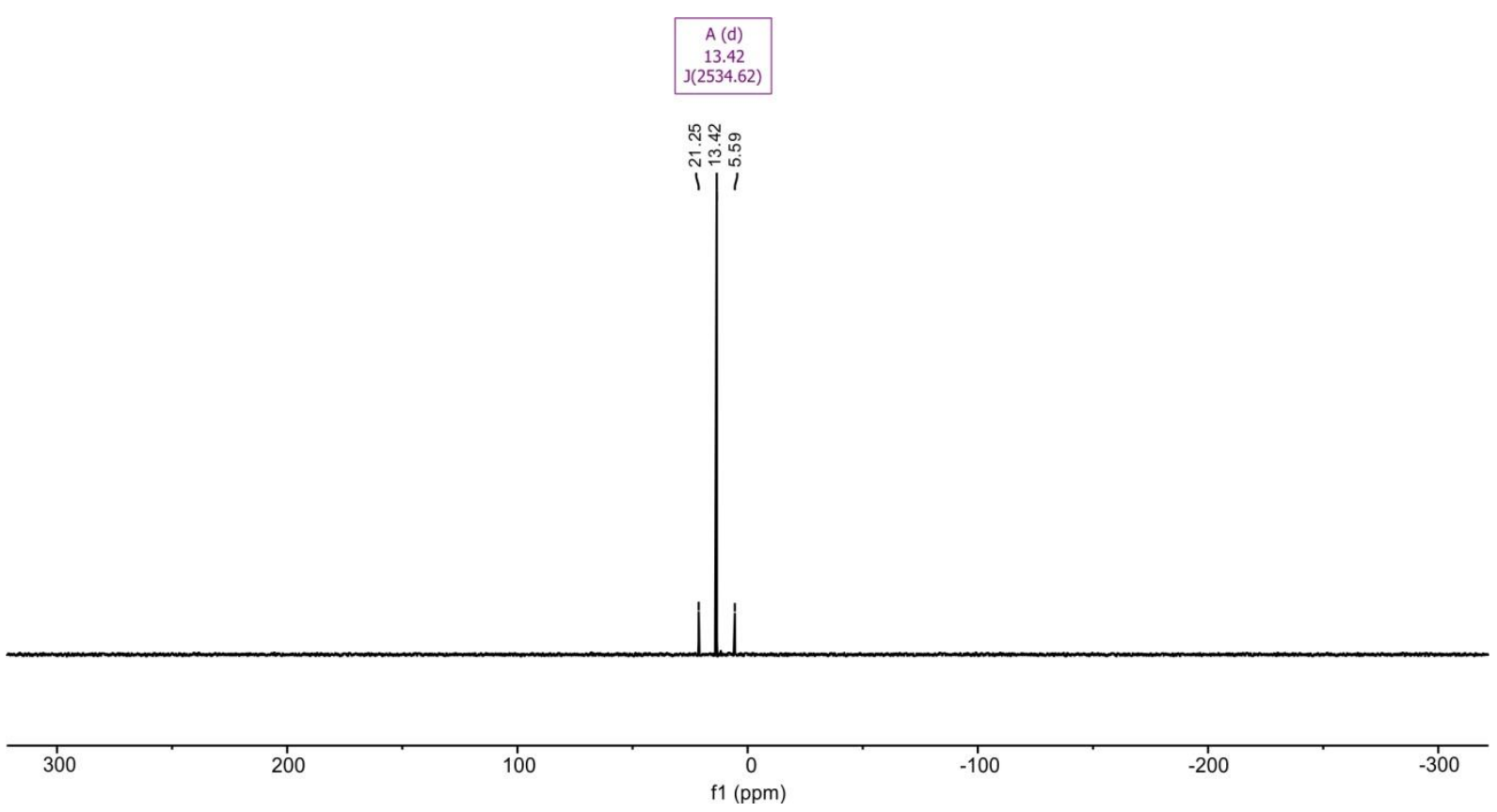

Figure S16. $161.8 \mathrm{MHz}^{31} \mathrm{P} \mathrm{NMR}$ of 2 in $\mathrm{CDCl}_{3}$. 


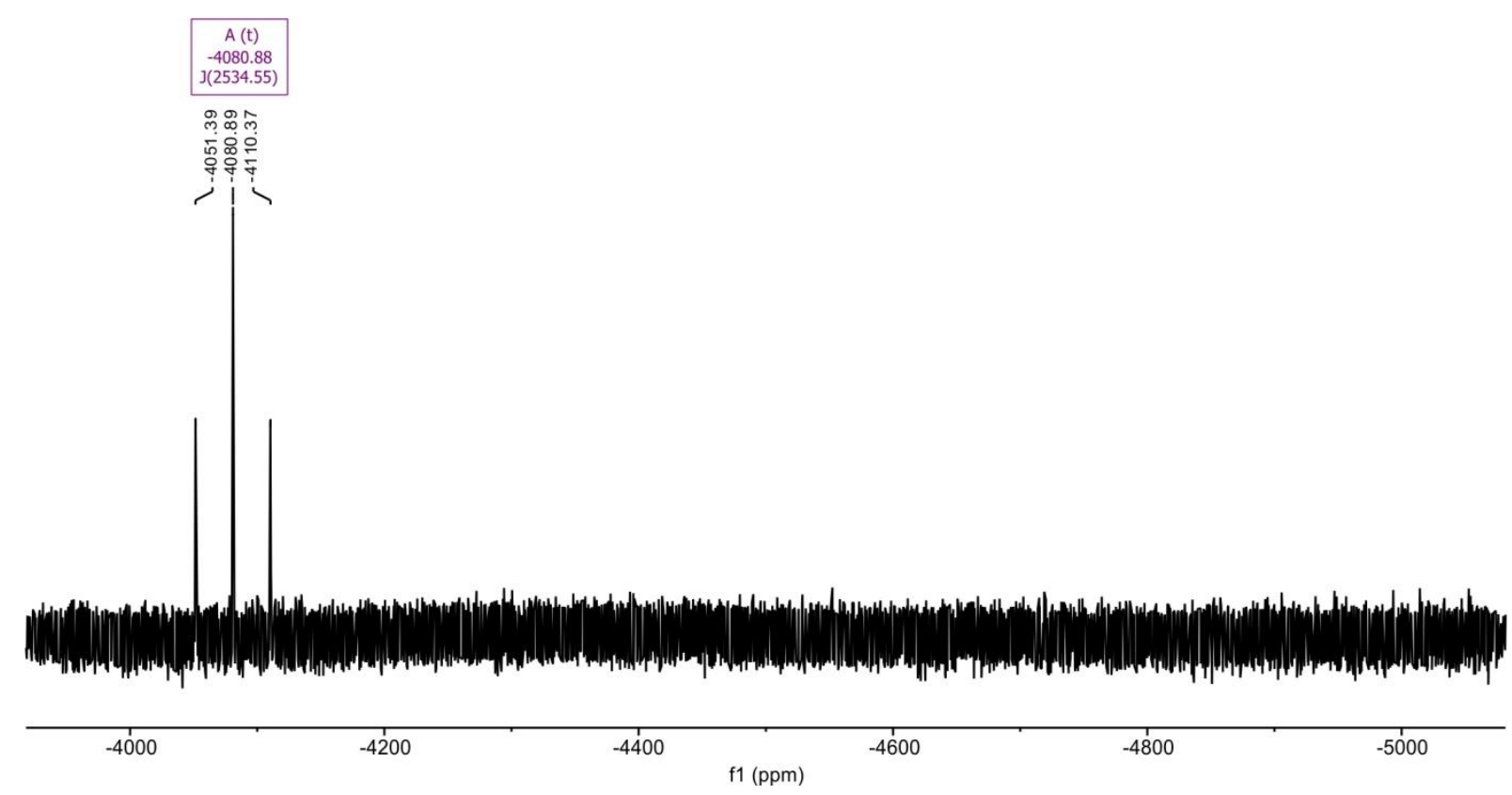

Figure S17. 85.6 MHz ${ }^{195} \mathrm{Pt} \mathrm{NMR}$ of 2 in $\mathrm{CDCl}_{3}$.

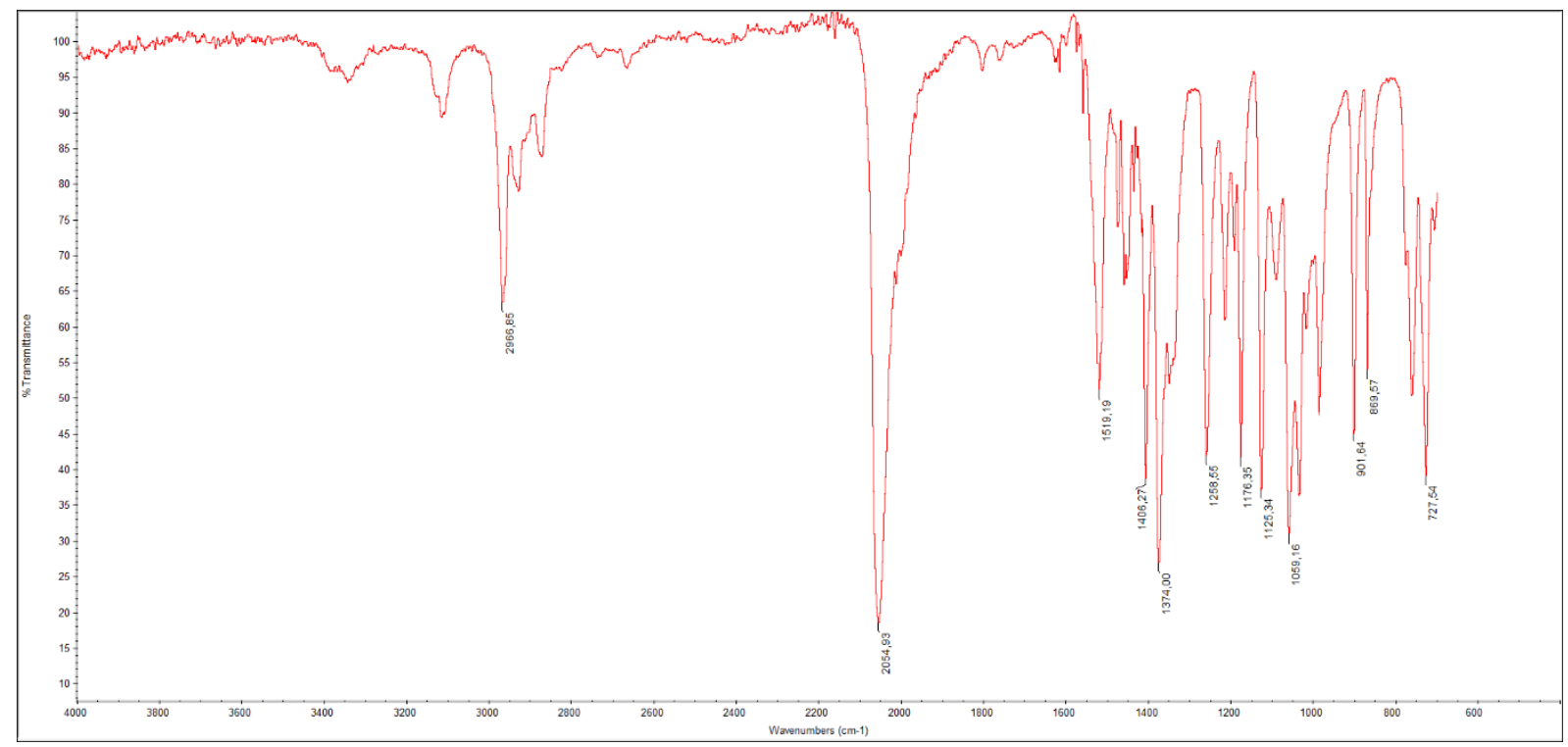

Figure S18. ATR IR of a solid sample of 2. 
Synthesis of $\left[\mathrm{Pt}(\right.$ bodipy $)\left(\right.$ triazolate $\left.\left.^{\mathrm{CF3}, \mathrm{COOEt}}\right)\left(\mathrm{PEt}_{3}\right)_{2}\right] 5$<smiles>CC[P+](N)(CC)[PH](N)(CC)CC</smiles>

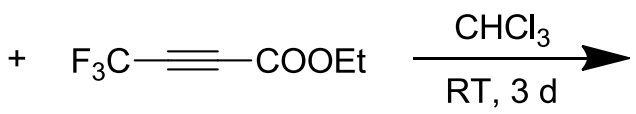<smiles>CCC[Pb]P</smiles>
$664.38 \mathrm{~g} / \mathrm{mol}$<smiles></smiles>

$\mathrm{C}_{27} \mathrm{H}_{41} \mathrm{BF}_{5} \mathrm{~N}_{5} \mathrm{O}_{2} \mathrm{P}_{2} \mathrm{Pt}$ $830.48 \mathrm{~g} / \mathrm{mol}$

Scheme S2. Synthesis of 5.

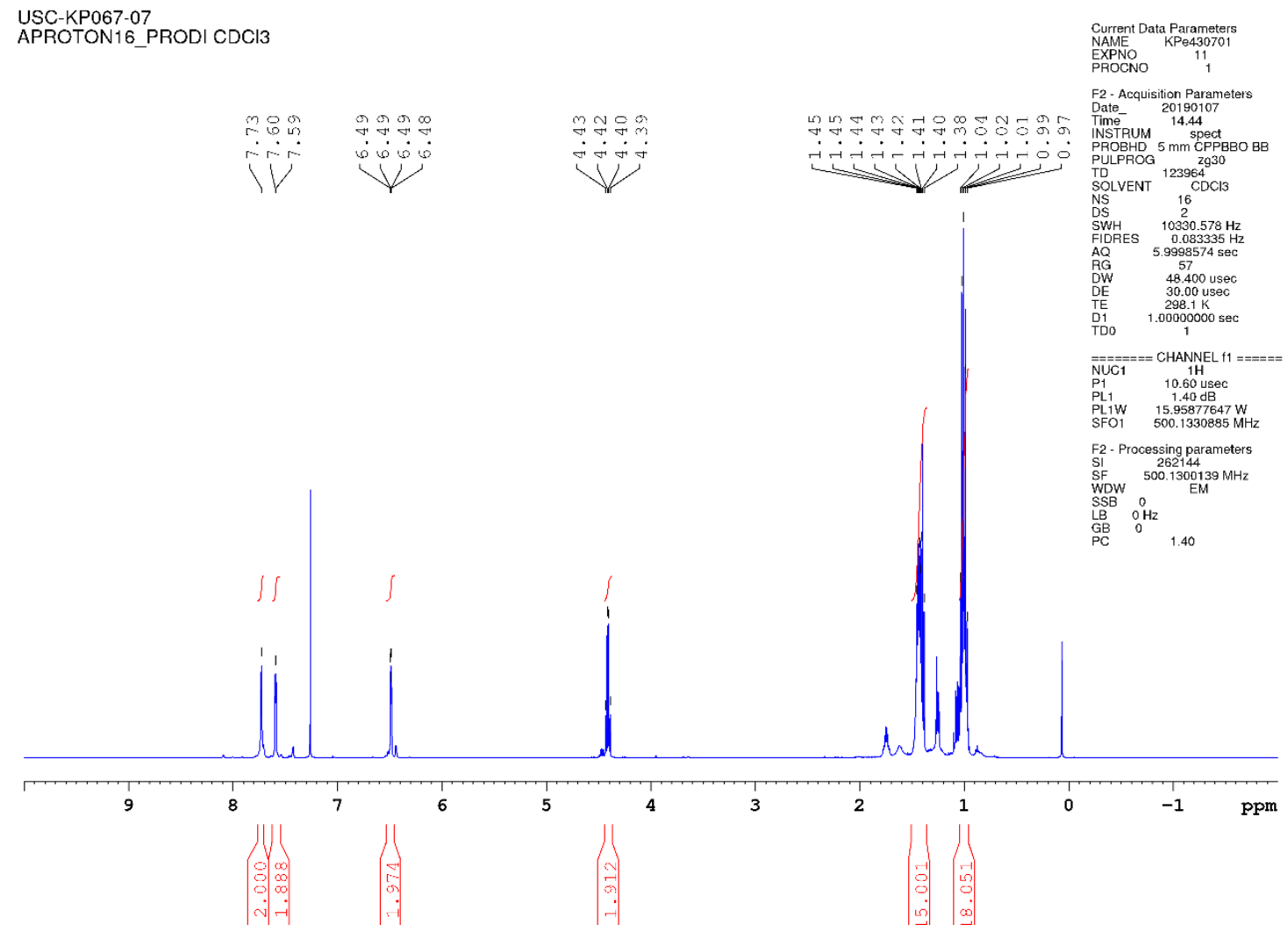

Figure $\mathbf{S 1 9} .500 \mathrm{MHz}{ }^{1} \mathrm{H}$ NMR of 5 in $\mathrm{CDCl}_{3}$. 
USC-KP067-07

AB11ZG PRODI CDCl3

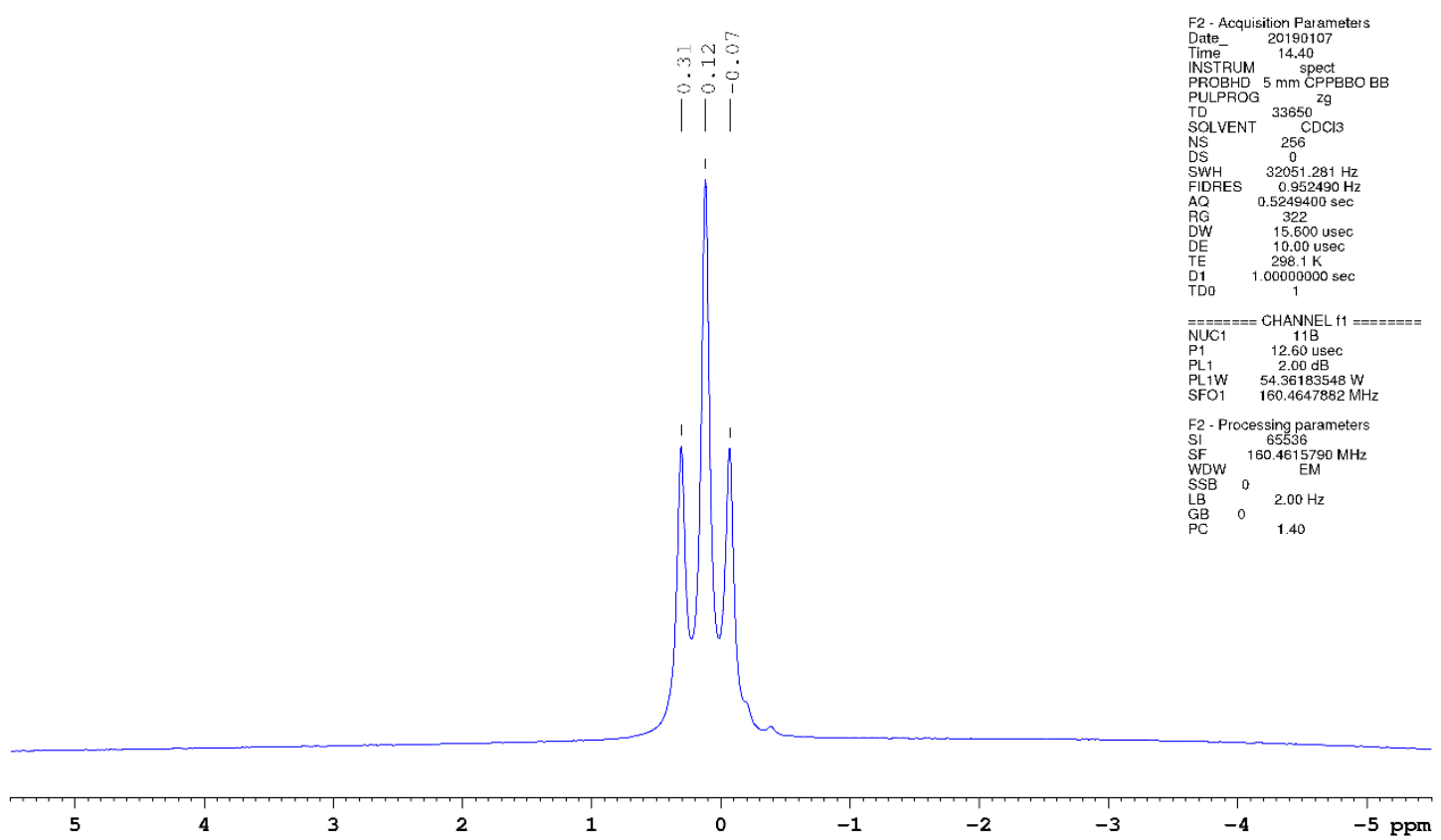

Figure S20. $160.5 \mathrm{MHz}{ }^{11} \mathrm{~B} \mathrm{NMR}$ of 5 in $\mathrm{CDCl}_{3}$.

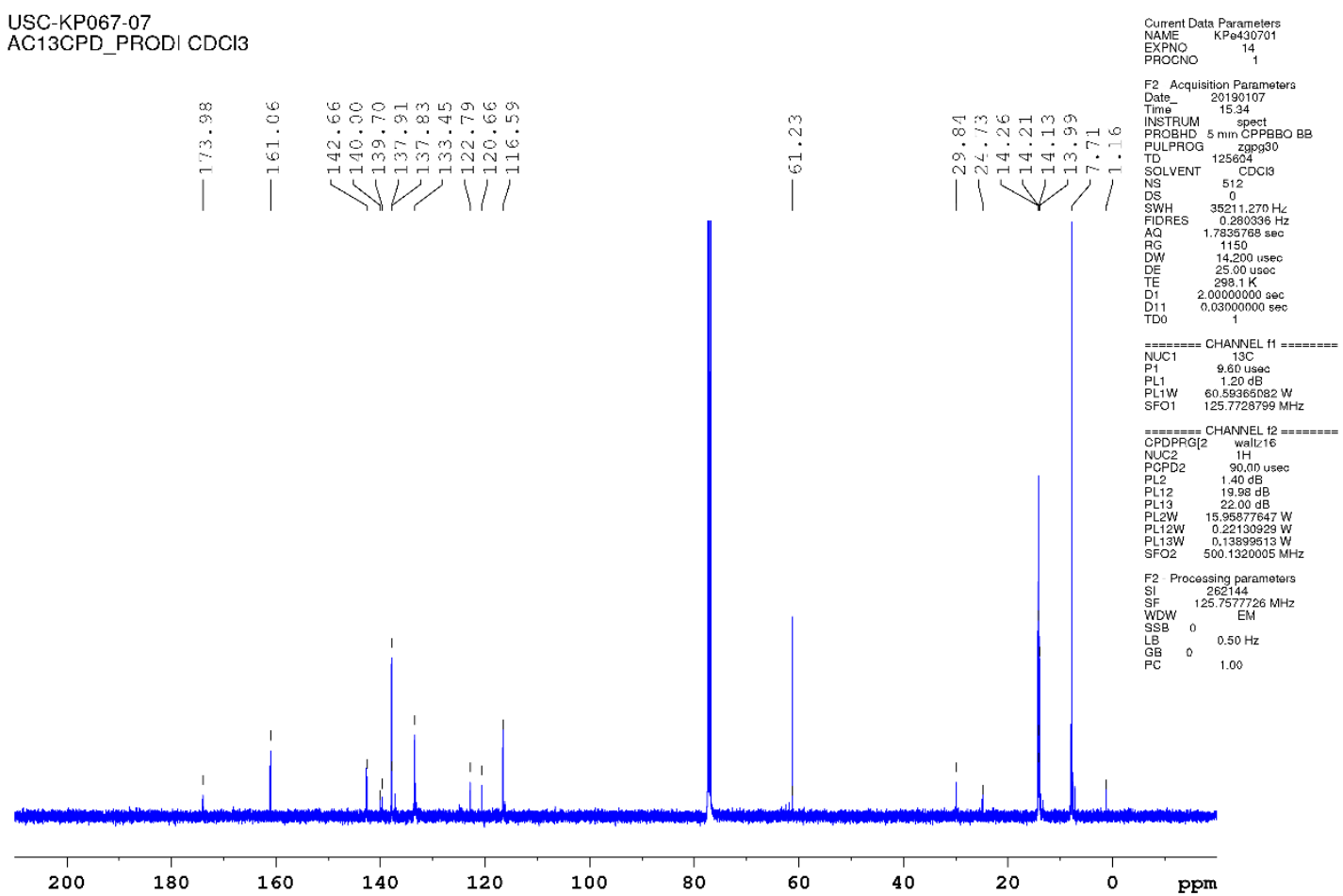

Figure S21. $125 \mathrm{MHz}{ }^{13} \mathrm{C}$ NMR of 5 in $\mathrm{CDCl}_{3}$. 


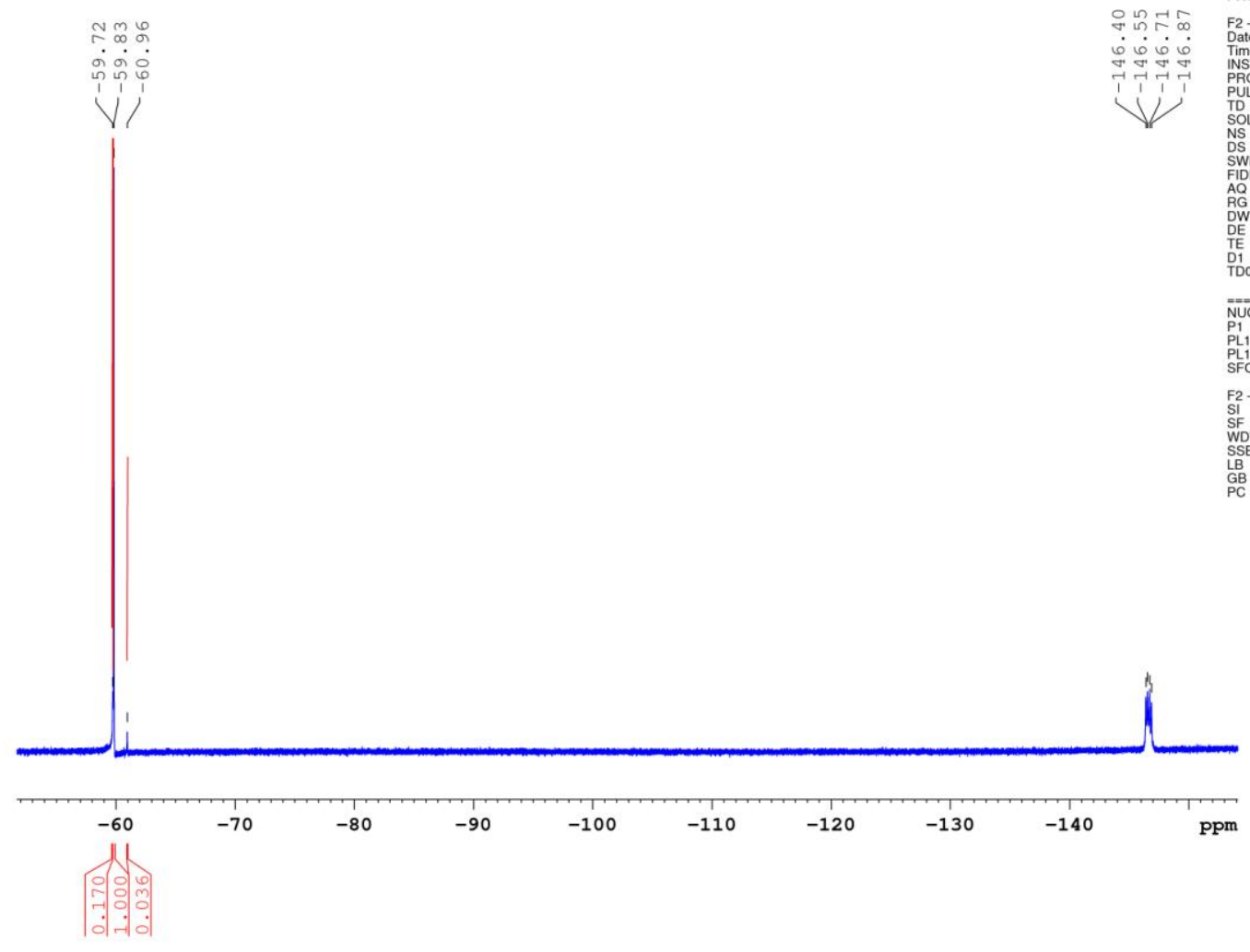

Figure S22. $188 \mathrm{MHz}{ }^{19} \mathrm{~F}$ NMR of 5 in $\mathrm{CDCl}_{3}$.

guest Peng
USC-KPo67-07

AP31CPD CDCl3 \{E:IBruker|Topspin\} User 43

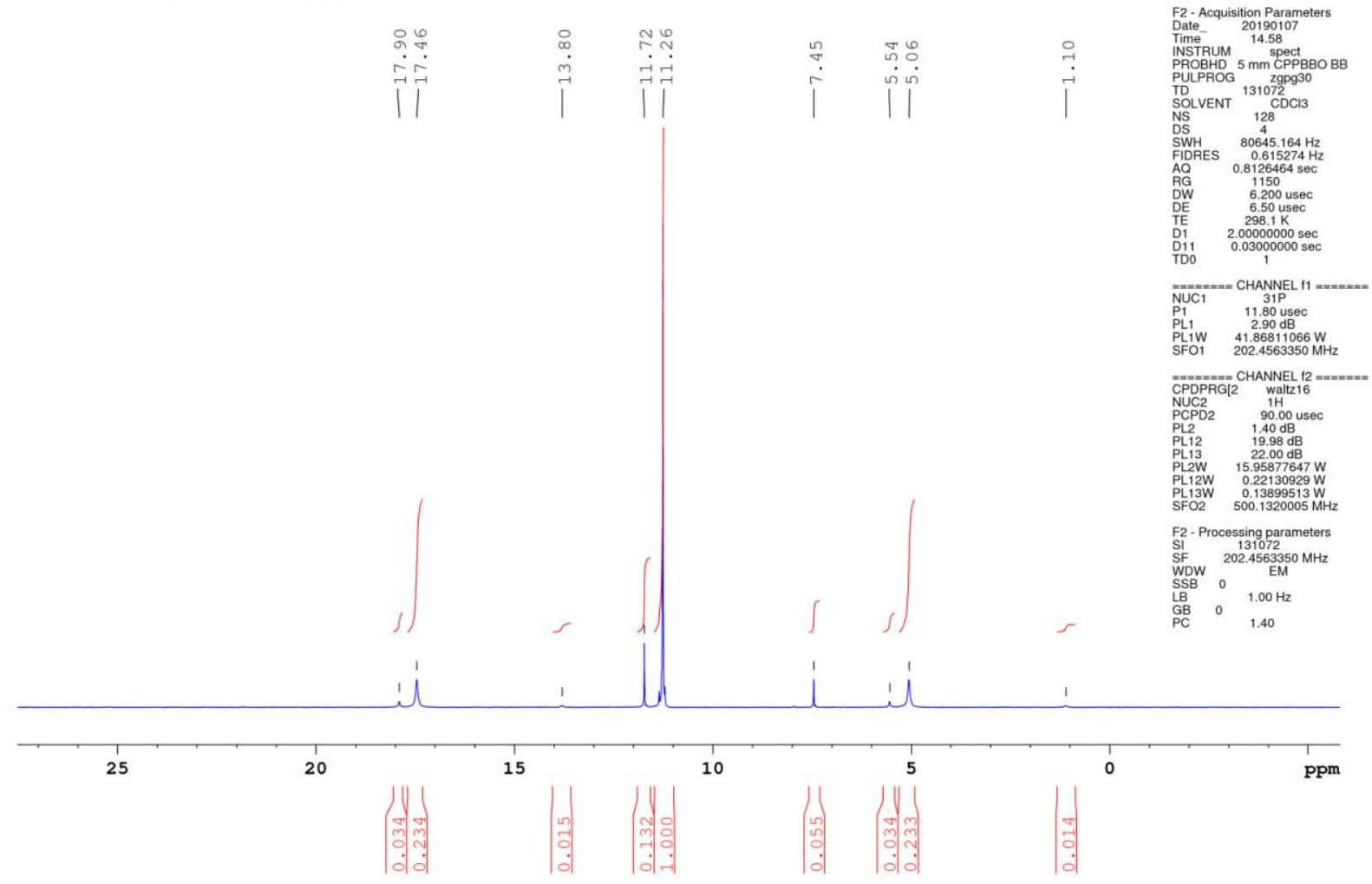

Figure S23. $202.5 \mathrm{MHz}{ }^{31} \mathrm{P} \mathrm{NMR}$ of 5 in $\mathrm{CDCl}_{3}$. 


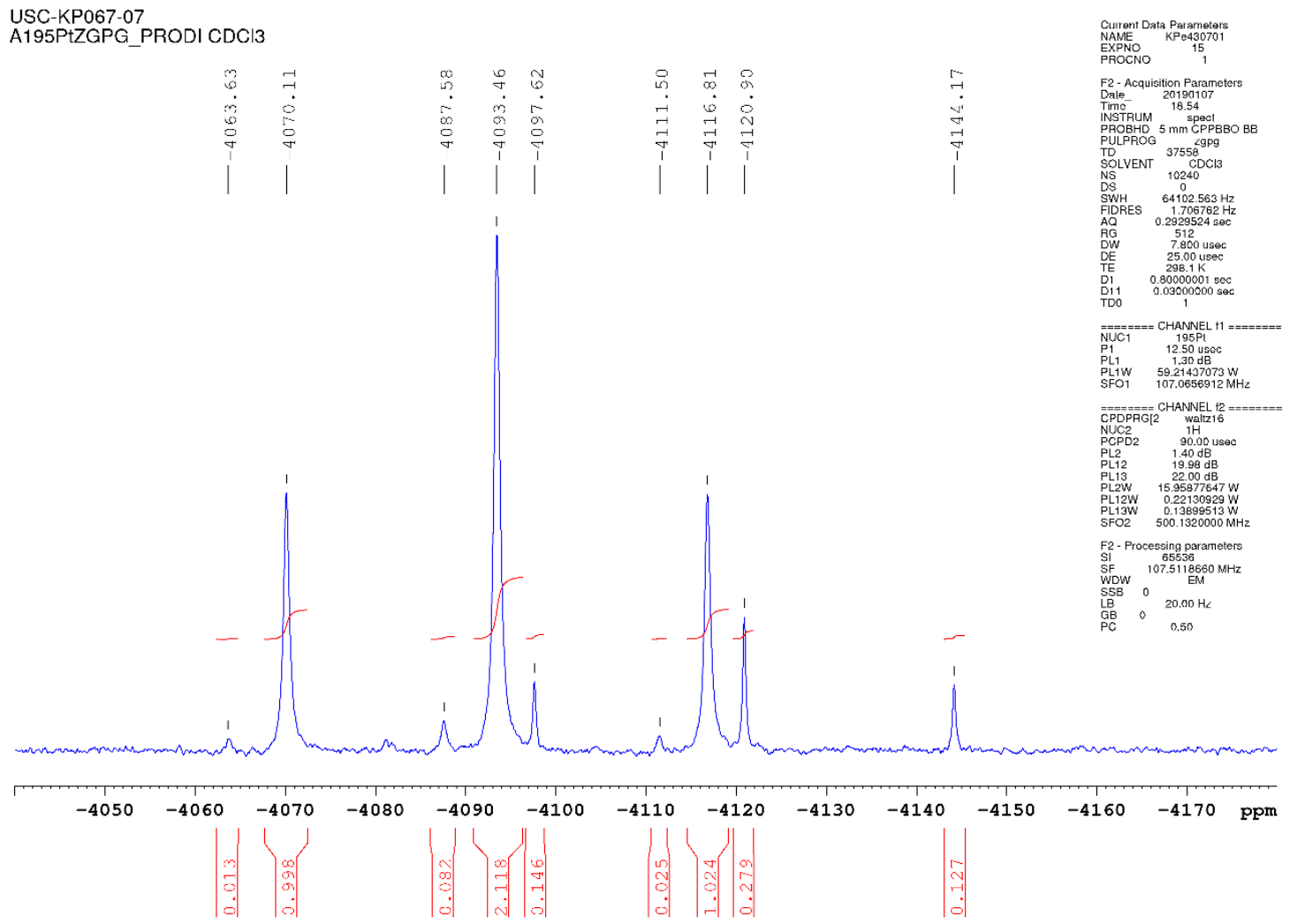

Figure S24. $107.5 \mathrm{MHz}{ }^{195} \mathrm{Pt} \mathrm{NMR}$ of $\mathbf{5}$ in $\mathrm{CDCl}_{3}$.

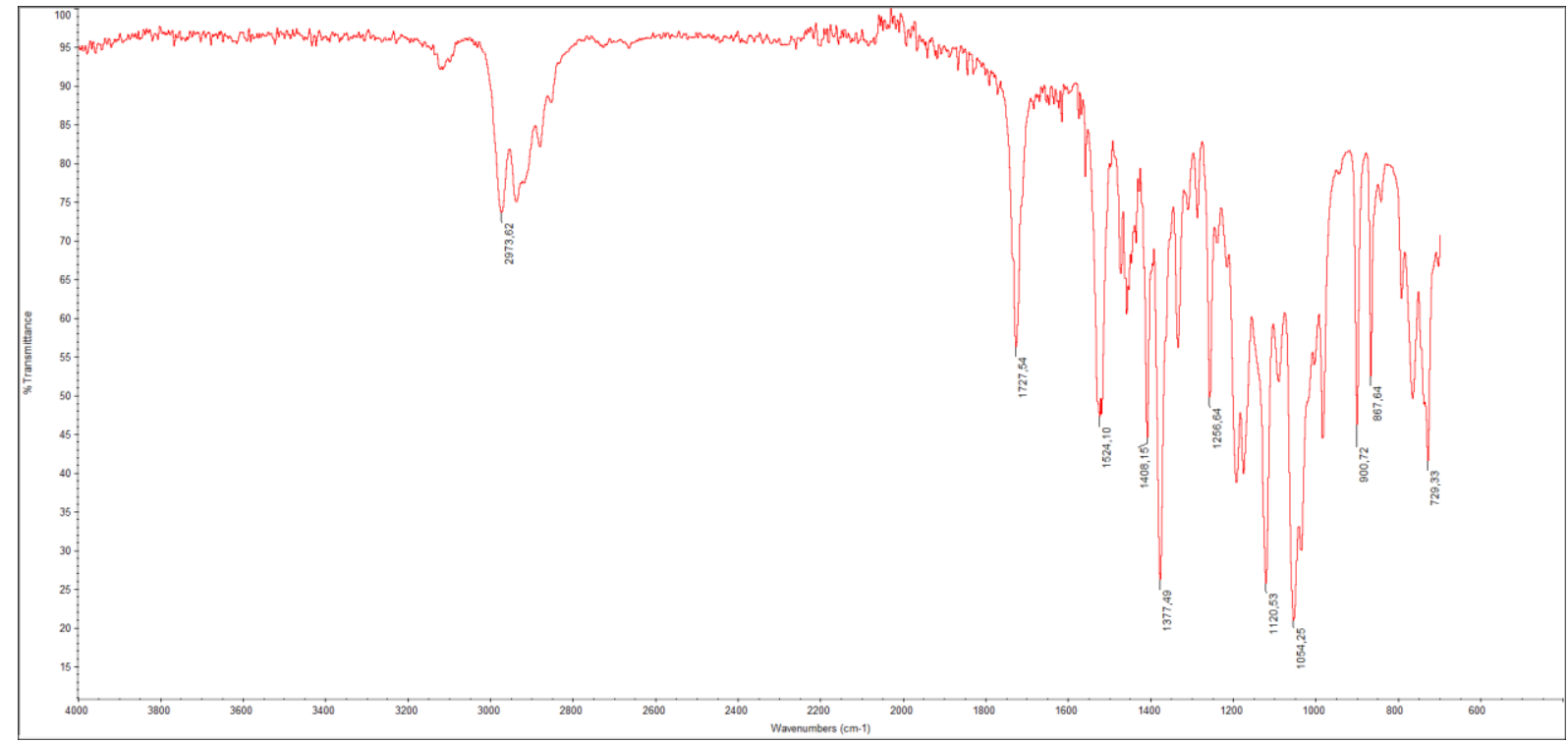

Figure S25. ATR IR of a solid sample of $\mathbf{5}$. 
Synthesis of $\left[\mathrm{Pt}(\right.$ bodipy $)\left(\right.$ triazolate $\left.\left.^{\mathrm{COOCH}, \mathrm{COOCH}}\right)\left(\mathrm{PEt}_{3}\right)_{2}\right] 6$<smiles></smiles>

$\mathrm{C}_{21} \mathrm{H}_{36} \mathrm{BF}_{2} \mathrm{~N}_{5} \mathrm{P}_{2} \mathrm{Pt}$ $664.38 \mathrm{~g} / \mathrm{mol}$

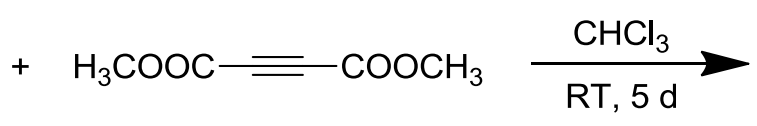

$$
\mathrm{C}_{6} \mathrm{H}_{6} \mathrm{O}_{4}
$$

$142.11 \mathrm{~g} / \mathrm{mol}$<smiles>COC(=O)c1n[nH]nc1C(=O)OC(C)=O</smiles><smiles></smiles>

$\mathrm{C}_{27} \mathrm{H}_{42} \mathrm{BF}_{2} \mathrm{~N}_{5} \mathrm{O}_{4} \mathrm{P}_{2} \mathrm{Pt}$ $806.49 \mathrm{~g} / \mathrm{mol}$

Scheme S3. Synthesis of 6.

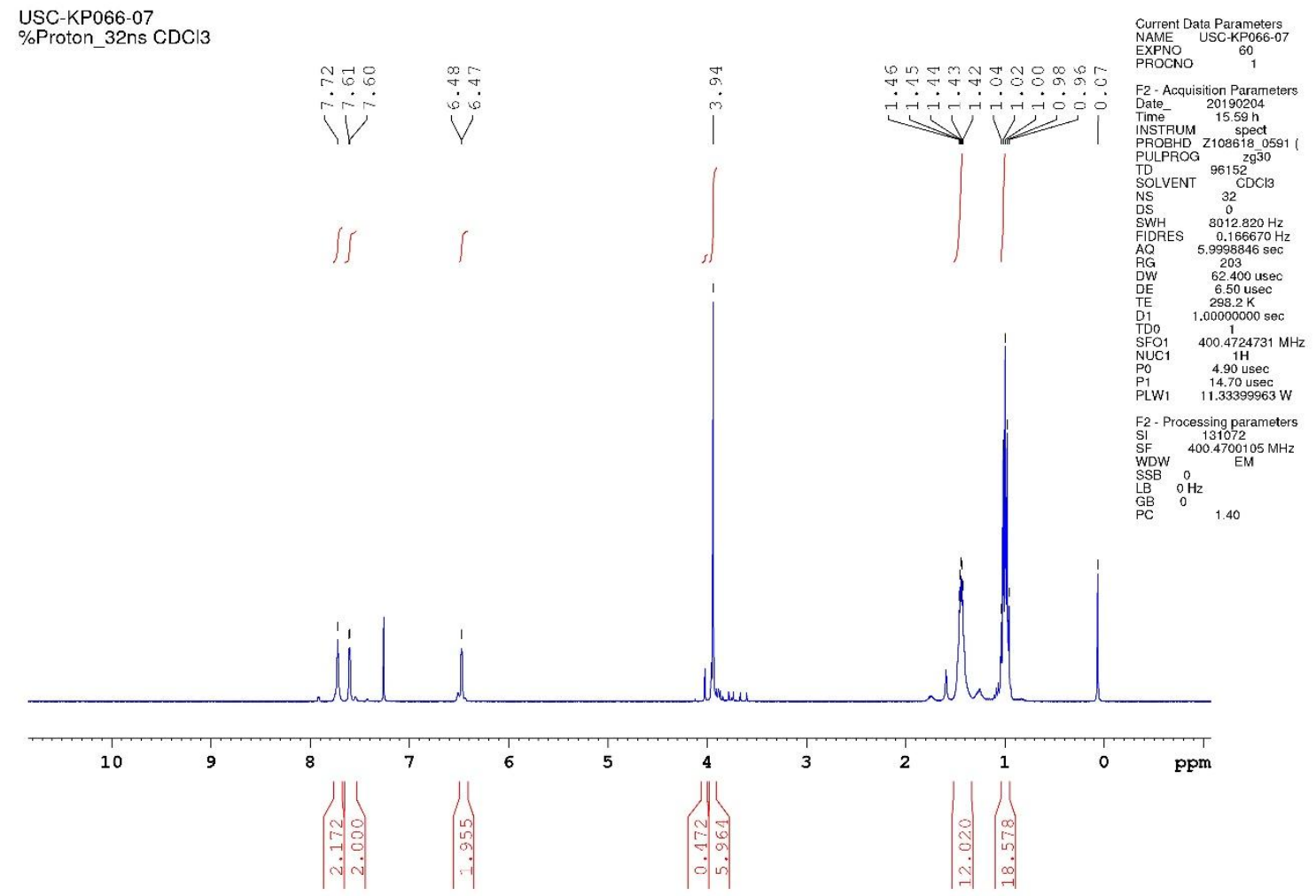

Figure S26. $400 \mathrm{MHz}{ }^{1} \mathrm{H}$ NMR of 6 in $\mathrm{CDCl}_{3}$. 


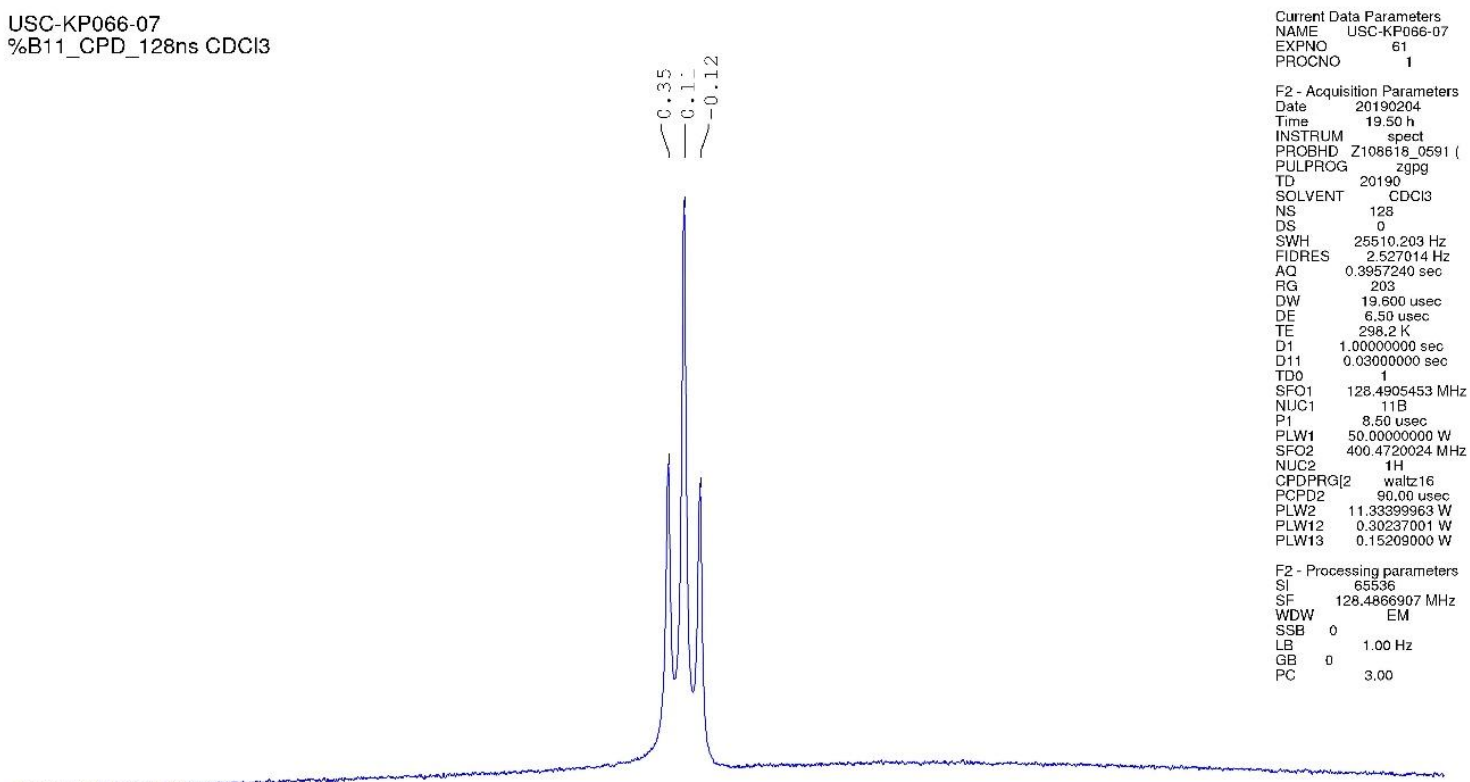

Figure S27. $128.5 \mathrm{MHz}{ }^{11} \mathrm{~B} \mathrm{NMR}$ of 6 in $\mathrm{CDCl}_{3}$.

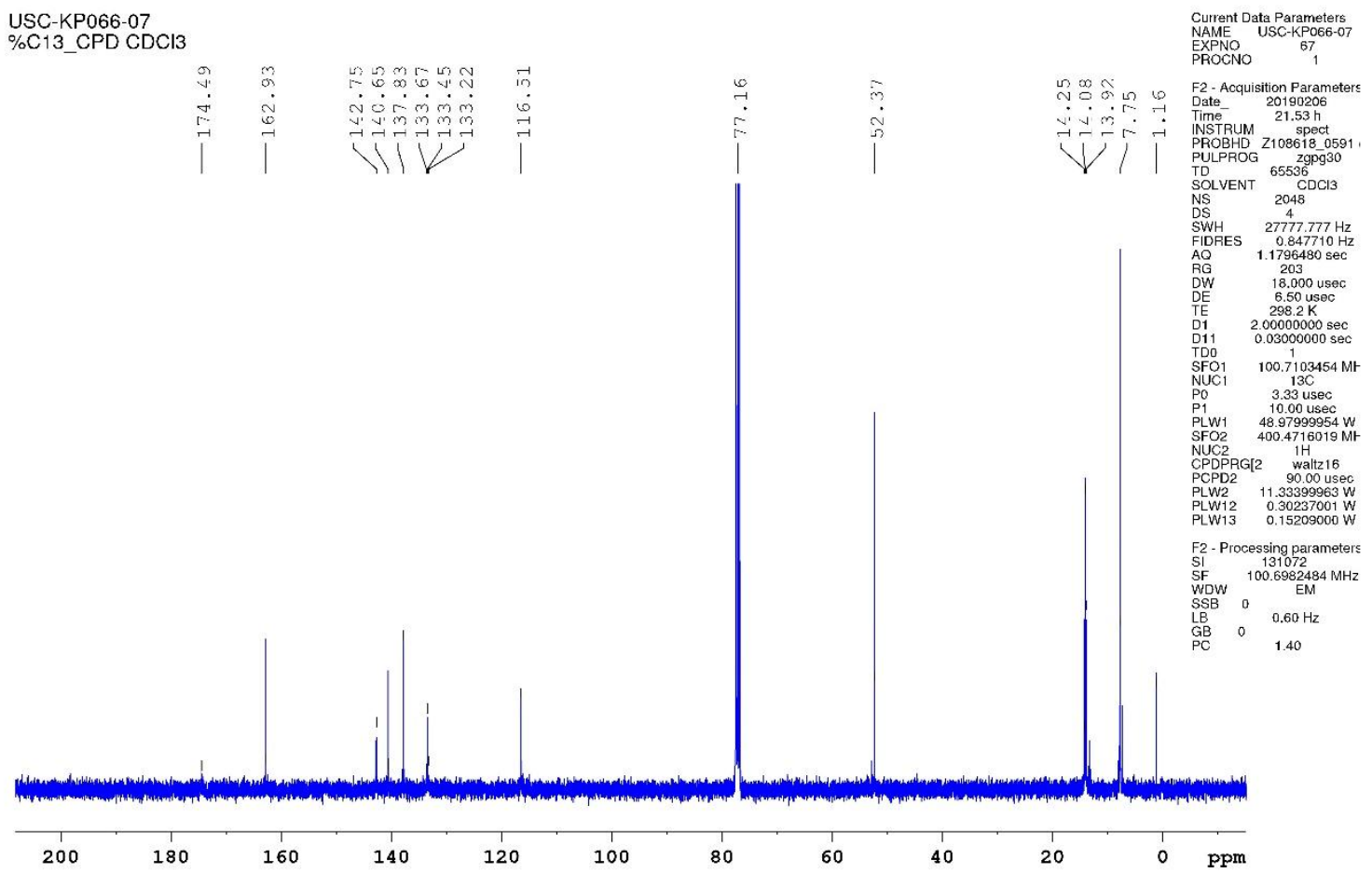

Figure S28. $100.7 \mathrm{MHz}{ }^{13} \mathrm{C} \mathrm{NMR}$ of 6 in $\mathrm{CDCl}_{3}$. 


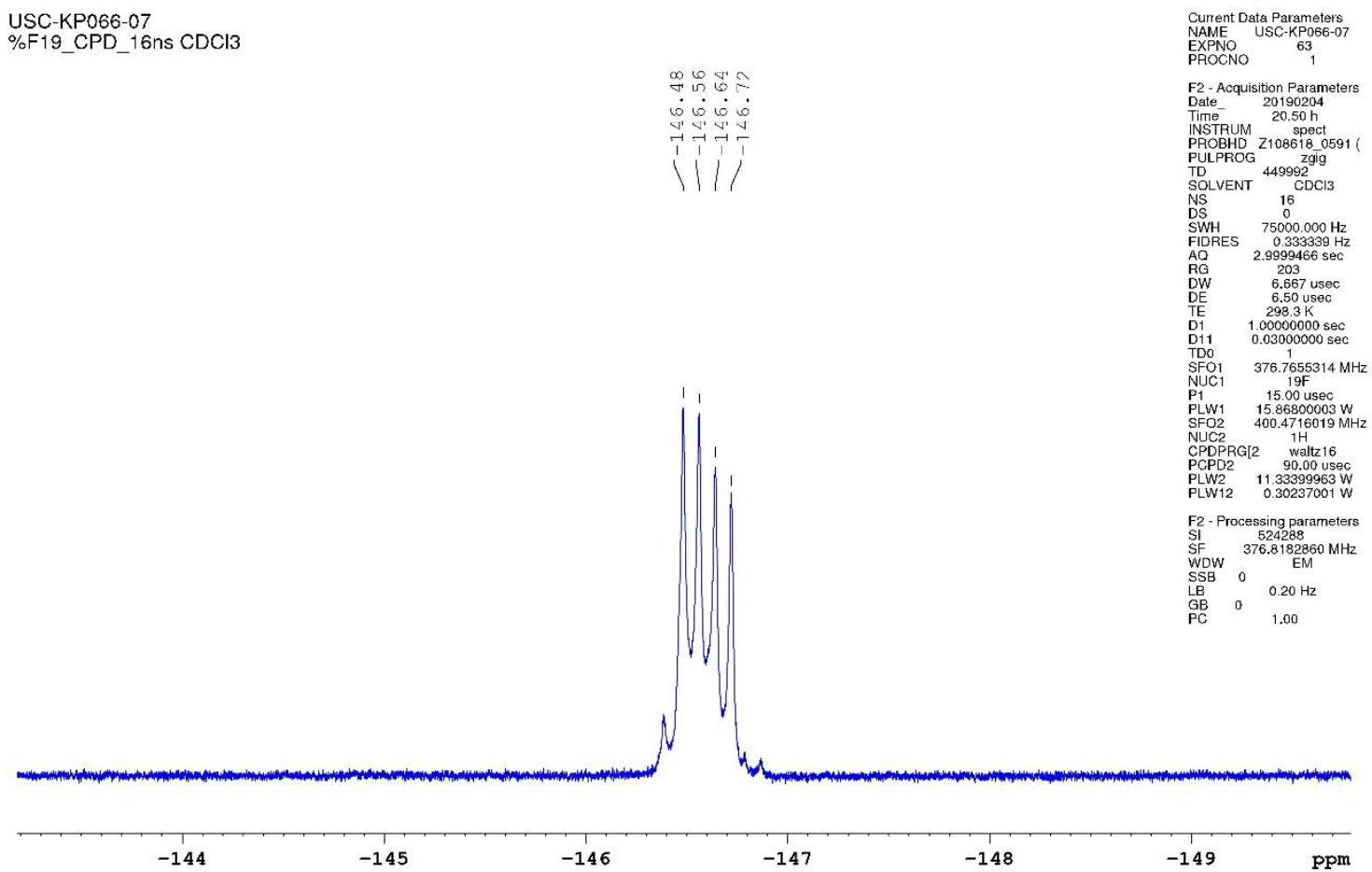

Figure S29. $376.8 \mathrm{MHz}{ }^{19} \mathrm{~F}$ NMR of 6 in $\mathrm{CDCl}_{3}$.

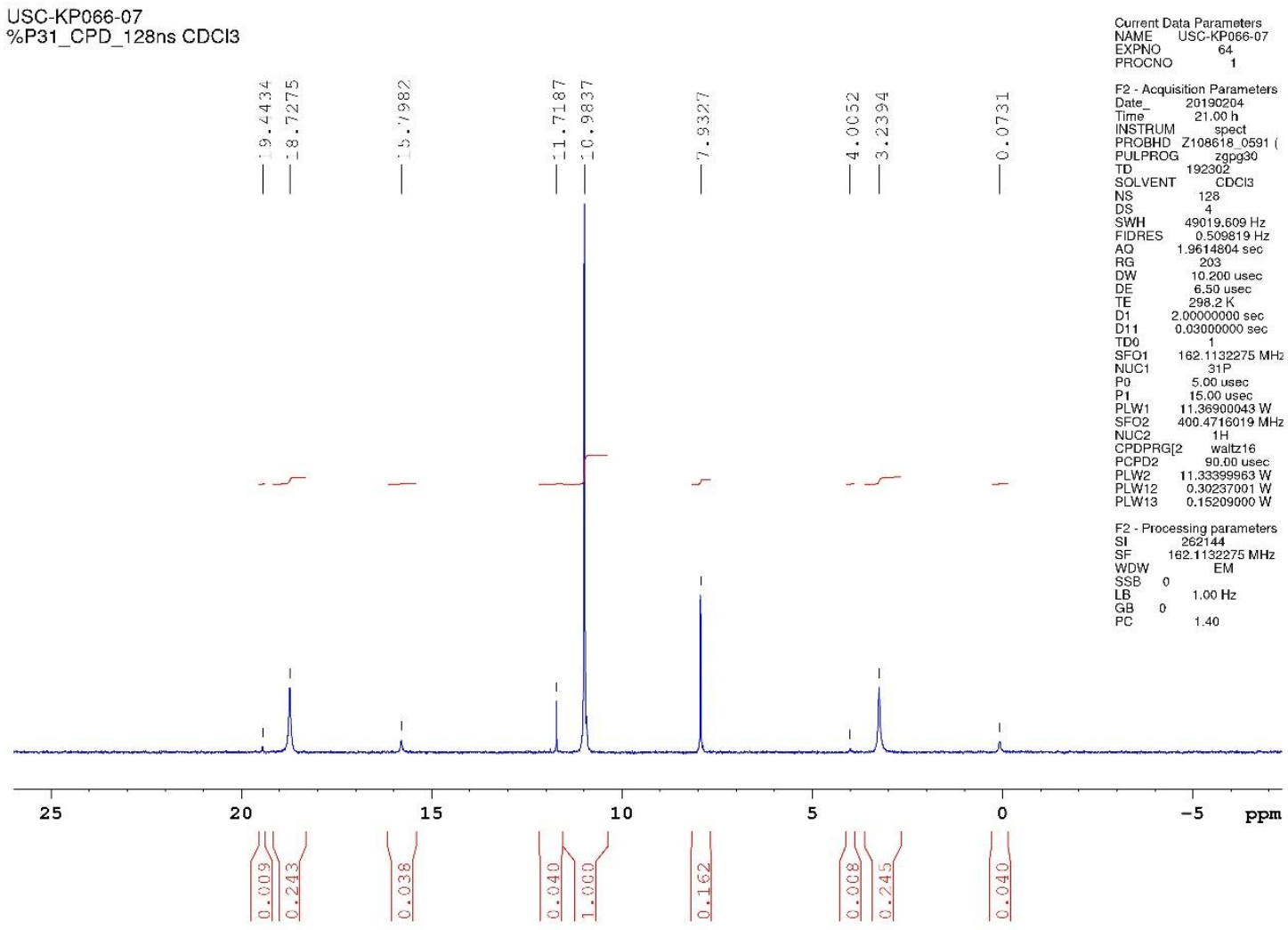

Figure S30. $162 \mathrm{MHz}^{31} \mathrm{P} \mathrm{NMR}$ of 6 in $\mathrm{CDCl}_{3}$. 


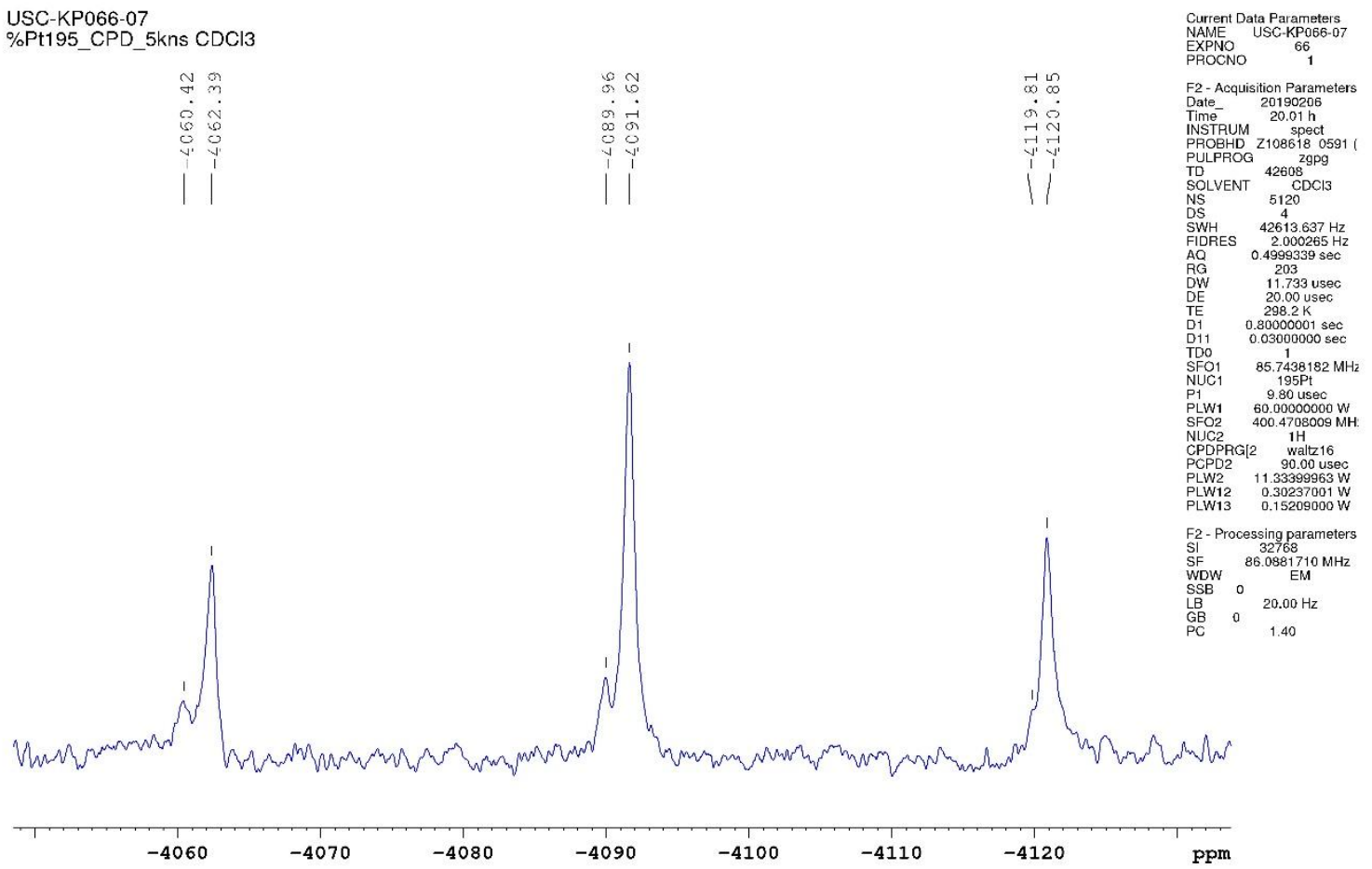

Figure S31. 86.1 MHz ${ }^{195} \mathrm{Pt} \mathrm{NMR}$ of $\mathbf{6}$ in $\mathrm{CDCl}_{3}$.

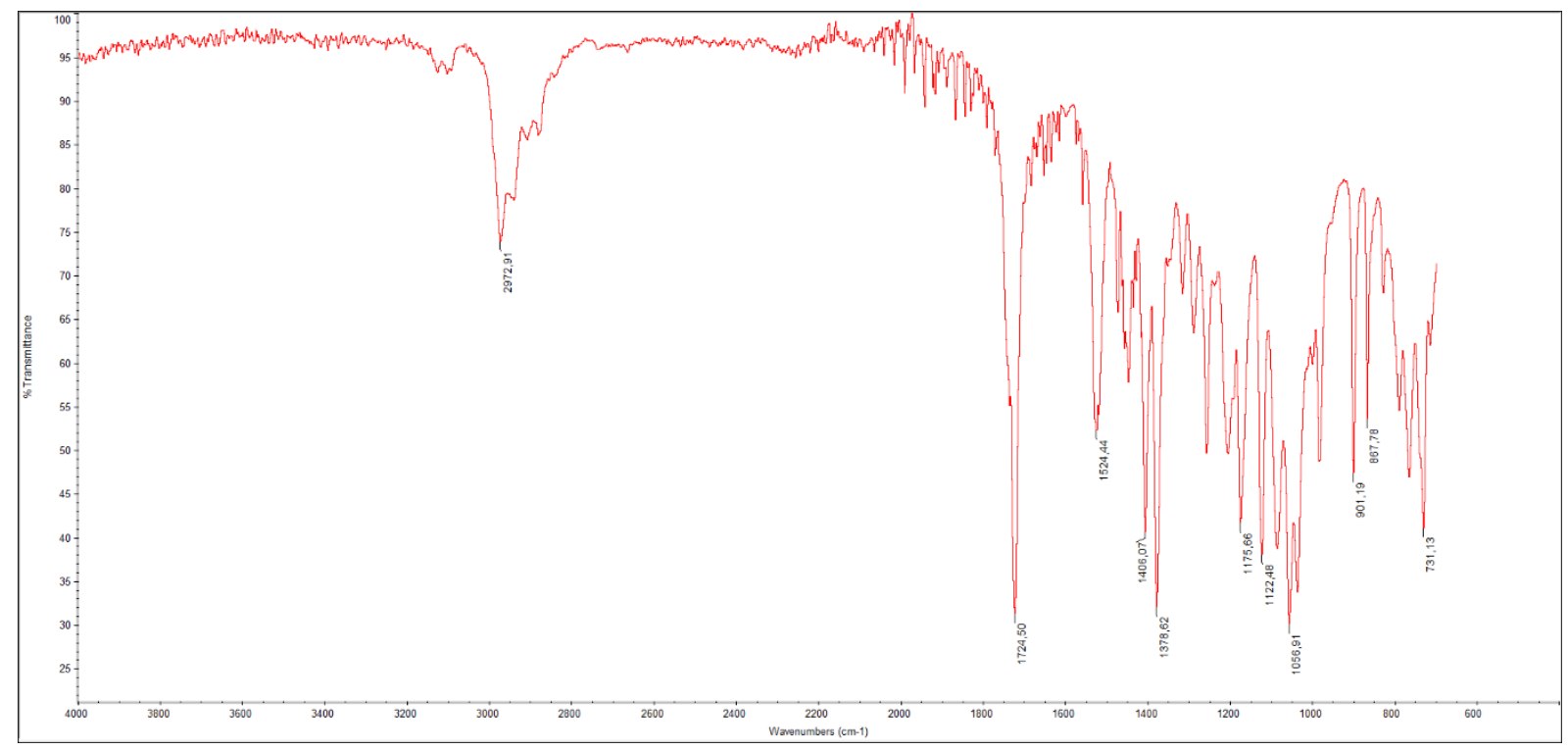

Figure S32. ATR IR of a solid sample of $\mathbf{6}$. 